Discrete Comput Geom 34:181-229 (2005)

DOI: $10.1007 / \mathrm{s} 00454-005-1176-0$

\title{
Chiral Polyhedra in Ordinary Space, $\mathbf{I I}^{*}$
}

\author{
Egon Schulte \\ Department of Mathematics, Northeastern University, \\ Boston, MA 02115, USA \\ schulte@neu.edu
}

With best wishes to Branko Grünbaum for his 75 th birthday

\begin{abstract}
A chiral polyhedron has a geometric symmetry group with two orbits on the flags, such that adjacent flags are in distinct orbits. Part I of the paper described the discrete chiral polyhedra in ordinary euclidean space $\mathbb{E}^{3}$ with finite skew faces and finite skew vertex-figures; they occur in infinite families and are of types $\{4,6\},\{6,4\}$ and $\{6,6\}$. Part II completes the enumeration of all discrete chiral polyhedra in $\mathbb{E}^{3}$. There exist several families of chiral polyhedra of types $\{\infty, 3\}$ and $\{\infty, 4\}$ with infinite, helical faces. In particular, there are no discrete chiral polyhedra with finite faces in addition to those described in Part I.
\end{abstract}

\section{Introduction}

The present paper continues the study of chiral polyhedra in ordinary euclidean space $\mathbb{E}^{3}$ begun in [24]. As polyhedra we permit discrete "polyhedra-like" structures in $\mathbb{E}^{3}$ with finite or infinite, planar or skew, polygonal faces or vertex-figures; more precisely, a polyhedron in $\mathbb{E}^{3}$ is a discrete three-dimensional faithful realization of an abstract regular polytope of rank 3 (see Chapter 5 of [20]). A polyhedron is (geometrically) chiral if its geometric symmetry group has exactly two orbits on the flags, such that adjacent flags are in distinct orbits. Chiral polyhedra are nearly regular polyhedra; recall that a (geometrically) regular polyhedron has a geometric symmetry group with just one orbit on the flags.

* This research was supported, in part, by NSA Grant H98230-04-1-0116, and was done, in part, while the author visited I.H.E.S. in Bures-sur-Yvette, France, for two months in 2002. The author thanks I.H.E.S. for the hospitality. 
The discrete regular polyhedra in $\mathbb{E}^{3}$ were completely enumerated by Grünbaum [11] and Dress [8], [9]; see Section 7E of [20] (or [19]) for a quick method of arriving at the full characterization, as well as for presentations of the symmetry groups.

In Part I we described a complete classification of the discrete chiral polyhedra with finite skew faces and finite skew vertex-figures in $\mathbb{E}^{3}$ [24]. There are three integer-valued two-parameter families of chiral polyhedra of this kind for each type $\{4,6\},\{6,4\}$ or $\{6,6\}$; some infinite families split further into several smaller subfamilies. Moreover, there are no chiral polyhedra in $\mathbb{E}^{3}$ which are finite.

The present Part II completes the enumeration of the discrete chiral polyhedra in $\mathbb{E}^{3}$. All chiral polyhedra not described in Part I have infinite, helical faces and occur again in infinite families; their types are $\{\infty, 3\}$ or $\{\infty, 4\}$, and their faces are helices over triangles or squares. We discuss their geometry and combinatorics in detail in Sections 4-8. The foundations are laid in Section 3, where we also prove that a chiral polyhedron cannot have finite planar faces or vertex-figures or an affinely reducible symmetry group; in effect, this shows that Part I actually enumerated all the chiral polyhedra in $\mathbb{E}^{3}$ with finite faces. Basic terminology and results are reviewed in Section 2 (following [20] and [24]).

\section{Basic Notions}

Although our main interest is in geometric polyhedra, we begin with a brief review of the underlying abstract theory (see Chapter 2 of [20]). Recall that an abstract polyhedron $\mathcal{P}$ is a partially ordered set of rank 3 . For $j=0,1$ or 2 , we call its $j$-faces vertices, edges and facets, respectively. When there is no possibility of confusion, we use the term "face" to mean "2-face" (facet). The flags of $\mathcal{P}$ each contain one vertex, one edge and one facet (as well as the minimal face $F_{-1}$ and maximal face $F_{3}$, usually omitted from the notation).

A polyhedron $\mathcal{P}$ is chiral if its (automorphism) group $\Gamma(\mathcal{P})$ has two orbits on the flags such that adjacent flags are in distinct orbits. Let $\Phi:=\left\{F_{0}, F_{1}, F_{2}\right\}$ be a fixed or base flag, and let $F_{j}^{\prime}$, with $j=0,1,2$, denote the $j$-face with $F_{j-1}<F_{j}^{\prime}<F_{j+1}$ and $F_{j}^{\prime} \neq F_{j}$. If $\mathcal{P}$ is chiral, then $\Gamma(\mathcal{P})$ is generated by distinguished generators $\sigma_{1}, \sigma_{2}$ (with respect to $\Phi$ ), where $\sigma_{1}$ fixes $F_{2}$ and cyclically permutes the vertices and edges of $F_{2}$ such that $F_{1} \sigma_{1}=F_{1}^{\prime}$, and $\sigma_{2}$ fixes $F_{0}$ and cyclically permutes the vertices and edges in the vertexfigure at $F_{0}$ such that $F_{2} \sigma_{2}=F_{2}^{\prime}$. A chiral polyhedron occurs in two enantiomorphic forms (see [25] and [26]); these correspond to the two (conjugacy classes of) pairs of distinguished generators of $\Gamma(\mathcal{P})$, namely the pair $\sigma_{1}, \sigma_{2}$ determined by the original base flag $\Phi$, and the pair $\sigma_{1} \sigma_{2}^{2}, \sigma_{2}^{-1}$ determined by the adjacent flag $\Phi^{2}:=\left\{F_{0}, F_{1}, F_{2}^{\prime}\right\}$.

A polyhedron $\mathcal{P}$ is regular if $\Gamma(\mathcal{P})$ is transitive on its flags. Then $\Gamma(\mathcal{P})$ is generated by distinguished generators $\rho_{0}, \rho_{1}, \rho_{2}$ (with respect to $\Phi$ ), where $\rho_{j}$ keeps all but the $j$-face of a chosen base flag $\Phi$ fixed. The elements $\sigma_{1}:=\rho_{0} \rho_{1}$ and $\sigma_{2}:=\rho_{1} \rho_{2}$ generate the (combinatorial) rotation subgroup $\Gamma^{+}(\mathcal{P})$ of $\Gamma(\mathcal{P})$. Now the two pairs of generators of $\Gamma^{+}(\mathcal{P})$ are conjugate in $\Gamma(\mathcal{P})$ under $\rho_{2}$, so the two enantiomorphic forms can be identified.

Let $\mathcal{P}$ be an abstract polyhedron, and let $\mathcal{P}_{j}$ denote its set of $j$-faces. A realization of $\mathcal{P}$ in $\mathbb{E}^{3}$ is a mapping $\beta: \mathcal{P}_{0} \rightarrow \mathbb{E}^{3}$ (see Section $5 \mathrm{~A}$ of [20]). Define $\beta_{0}:=\beta$ and $V_{0}:=V:=\mathcal{P}_{0} \beta$. Then $\beta$ recursively induces surjections $\beta_{j}: \mathcal{P}_{j} \rightarrow V_{j}$, for $j=1,2,3$, 
with $V_{j} \subset 2^{V_{j-1}}$ (the family of subsets of $V_{j-1}$ ) consisting of the elements

$$
F \beta_{j}:=\left\{G \beta_{j-1} \mid G \in \mathcal{P}_{j-1} \text { and } G \leqslant F\right\} \quad\left(F \in \mathcal{P}_{j}\right) .
$$

A realization $\beta$ is faithful if each $\beta_{j}$ is a bijection; otherwise, $\beta$ is degenerate. In a symmetric realization $\beta$, each automorphism of $\mathcal{P}$ induces an isometric permutation of the vertex-set $V$; this yields a representation of $\Gamma(\mathcal{P})$ as a group of euclidean isometries. The realization theory even of simple polyhedra can be rather complicated; see, for example, Section 5B of [20] or [22].

We mostly work with discrete and faithful realizations. In this case the vertices, edges and facets of $\mathcal{P}$ are in one-to-one correspondence with certain points, line segments and simple (finite or infinite) polygons in $\mathbb{E}^{3}$, and it is safe to identify a face of $\mathcal{P}$ and its image in $\mathbb{E}^{3}$. The resulting family of points, line segments and polygons is a (discrete) geometric polyhedron in $\mathbb{E}^{3}$ and is denoted by $P$; it is understood that $P$ inherits the partial ordering of $\mathcal{P}$, and when convenient $P$ will be identified with $\mathcal{P}$.

Recall that a geometric polyhedron $P$ in $\mathbb{E}^{3}$ is geometrically regular if its (geometric) symmetry group $G(P)$ is flag-transitive, and that $P$ is geometrically chiral if $G(P)$ has two orbits on the flags such that adjacent flags are in distinct orbits (see [24]). In the latter case the underlying abstract polyhedron $\mathcal{P}$ must be (combinatorially) chiral or regular. Note that there are geometric polyhedra which are not chiral but still have a symmetry group with only two orbits on the flags (see [15], [29] and [30]). For other classes of highly symmetric polyhedra see, for example, [13] and [14].

The structure results for abstract polyhedra carry over to geometric polyhedra as follows. Let $P$ be a discrete (geometrically) chiral or regular (geometric) polyhedron in $\mathbb{E}^{3}$. As before, let $\Phi:=\left\{F_{0}, F_{1}, F_{2}\right\}$ be a base flag of $P$, and let $F_{j}^{\prime}$, with $j=0,1,2$, be the $j$-face of $P$ with $F_{j-1}<F_{j}^{\prime}<F_{j+1}$ and $F_{j}^{\prime} \neq F_{j}$.

If $P$ is chiral, then $G:=G(P)$ is generated by distinguished generators $S_{1}, S_{2}$ (with respect to $\Phi)$, where $S_{1}$ fixes the base facet $F_{2}$ and cyclically permutes its vertices such that $F_{1} S_{1}=F_{1}^{\prime}$ (and thus $F_{0}^{\prime} S_{1}=F_{0}$ ), and $S_{2}$ fixes the base vertex $F_{0}$ and cyclically permutes the vertices in the vertex-figure at $F_{0}$ such that $F_{2} S_{2}=F_{2}^{\prime}$ (and thus $F_{1}^{\prime} S_{2}=F_{1}$ ). Then

$$
S_{1}^{p}=S_{2}^{q}=\left(S_{1} S_{2}\right)^{2}=I,
$$

the identity mapping, with $p, q$ given by the type $\{p, q\}$ of $P$ (see [7]). The involution $T:=S_{1} S_{2}$ interchanges the two vertices in $F_{1}$ as well as the two faces meeting at $F_{1}$.

If $P$ is regular, then $G(P)$ is generated by distinguished generators $R_{0}, R_{1}, R_{2}$ (with respect to $\Phi)$, where $R_{j}$ maps all but the $j$-face of $\Phi$ to itself. Each $R_{j}$ is a reflection in a point, line or plane. If $S_{1}:=R_{0} R_{1}$ and $S_{2}:=R_{1} R_{2}$, then the subgroup $G:=\left\langle S_{1}, S_{2}\right\rangle$ of $G(P)$ of index 2 has properties very similar to those of the group of a chiral polyhedron. Now $T:=S_{1} S_{2}=R_{0} R_{2}$, and $T$ again interchanges the two vertices of $F_{1}$ as well as the two faces meeting at $F_{1}$. For a regular polyhedron, the dimension vector

$$
\left(\operatorname{dim} R_{0}, \operatorname{dim} R_{1}, \operatorname{dim} R_{2}\right)
$$

records the dimensions of the reflection mirrors for $R_{0}, R_{1}, R_{2}$. See Section 7E of [20] for a complete enumeration of the regular polyhedra in $\mathbb{E}^{3}$ (see also [8], [9], [11] and [16]).

For emphasis, throughout the paper, $G$ denotes the subgroup $\left\langle S_{1}, S_{2}\right\rangle$ of $G(P)$, irrespective of whether $P$ is chiral or regular. Then $G=G(P)$ if $P$ is chiral, or $G$ is a subgroup of index 2 in $G(P)$ if $P$ is regular. 
We require the following lemma, which gives a useful necessary and sufficient condition for a polyhedron to be regular.

Lemma 2.1. Let $P$ be a discrete chiral or regular polyhedron in $\mathbb{E}^{3}$ with associated group $G=\left\langle S_{1}, S_{2}\right\rangle$. Let $\Phi=\left\{F_{0}, F_{1}, F_{2}\right\}$ be the corresponding base flag, and let $F_{j}^{\prime}$, for $j=0,1,2$, be the $j$-face associated with $\Phi$ as above. Then $P$ is regular if and only if there exists an isometry $R$ of $\mathbb{E}^{3}$ such that

$$
R^{-1} S_{2} R=S_{2}^{-1}, \quad R^{-1} T R=T, \quad F_{0} R=F_{0}, \quad F_{1} R=F_{1}, \quad F_{2} R=F_{2}^{\prime} .
$$

Proof. If $P$ is regular, then $R:=R_{2}$ has the required properties. Conversely, suppose that $R$ is an isometry acting in the way described. Then $R^{-1} G R=G$ because $G$ is generated by $S_{2}$ and $T$, and

$$
\left(F_{i} G\right) R=\left(F_{i} R\right) G=F_{i} G \text { or } F_{i}^{\prime} G
$$

according as $i=0,1$ or $i=2$. Since there is only one orbit on the $i$-faces for each $i$ (even when $i=2$ ), this implies that $R$ must indeed be a symmetry of $P$, which then takes $\Phi$ to the adjacent flag $\Phi^{2}=\left\{F_{0}, F_{1}, F_{2}^{\prime}\right\}$. Hence $P$ must be regular and $R=R_{2}$.

Note that Lemma 2.1 can be employed to shorten the proof of Theorem 3.1 of [24], which says that there are no finite chiral polyhedra in $\mathbb{E}^{3}$.

Geometrically chiral or regular polyhedra can be obtained by a variant of Wythoff's construction (see [6] or Section 5A of [20]). Let an abstract polyhedron $\mathcal{P}$ be chiral or regular, and let $G:=\left\langle S_{1}, S_{2}\right\rangle$ be a euclidean representation of $\Gamma(\mathcal{P})$ or $\Gamma^{+}(\mathcal{P})$, respectively. Each point $v$ which is fixed by $S_{2}$ can serve as the initial vertex of a realization $P$ with vertex-set $V=v G$. Its base vertex, base edge and base face are $v$, $v\langle T\rangle$ or $v\left\langle S_{1}\right\rangle$, respectively, and the other vertices, edges and faces are their images under $G$. In our applications, $P$ will generally be a geometric polyhedron. Note that, a priori, an abstract regular polyhedron can have a realization which is geometrically chiral.

Certain operations that can be applied to regular polyhedra have analogs that also apply to abstract chiral polyhedra and frequently to geometrically chiral polyhedra as well. Examples are the duality operation $\delta$ and the ( $2 n d$ ) facetting operation $\varphi_{2}$, yielding the generators for the groups of the dual polyhedron $\mathcal{P}^{*}:=\mathcal{P}^{\delta}$ or of $\mathcal{P}^{\varphi_{2}}$, respectively (see pp. 192 and 194 of [20]). If an abstract polyhedron $\mathcal{P}$ is chiral, then $\delta$ and $\varphi_{2}$ are given by

$$
\delta:\left(\sigma_{1}, \sigma_{2}\right) \mapsto\left(\sigma_{2}^{-1}, \sigma_{1}^{-1}\right) \quad \text { and } \quad \varphi_{2}:\left(\sigma_{1}, \sigma_{2}\right) \mapsto\left(\sigma_{1} \sigma_{2}^{-1}, \sigma_{2}^{2}\right) .
$$

When applied to the rotation subgroup $\Gamma^{+}(\mathcal{P})$ of a regular polyhedron $\mathcal{P}$, the new generators (on the right) become the distinguished generators for the rotation subgroups of the polyhedra $\mathcal{P}^{\delta}$ or $\mathcal{P}^{\varphi_{2}}$ of Section 7B of [20], respectively. If $\mathcal{P}$ is a chiral polyhedron, then moving from one enantiomorphic form to the other corresponds to applying the enantiomorphism operation

$$
\mu:\left(\sigma_{1}, \sigma_{2}\right) \mapsto\left(\sigma_{1} \sigma_{2}^{2}, \sigma_{2}^{-1}\right) .
$$


The operations $\delta, \varphi_{2}$ and $\mu$ are examples of mixing operations (see p. 83 of [20]) and carry over to geometric polyhedra.

Suppose $\mathcal{P}$ (or $P$ ) is a chiral polyhedron of type $\{p, q\}$ with $p$ and $q$ finite. For odd $q$, the geometric effect of $\varphi_{2}$ on $\mathcal{P}$ is as described on p. 195 of [20] (for regular polyhedra), so we do not repeat it here. However, in the present context we are only interested in the case when $q \geqslant 6$ is even, and specifically when $q=6$. Here it is helpful to think of $\mathcal{P}$ as a map on a surface $M$ (compare [2] and [7]), and of $\Gamma(\mathcal{P})$ as a group of homeomorphisms which preserves $\mathcal{P}$ and its order complex. The triangles in the order complex correspond to the flags of $\mathcal{P}$, so in particular we have a base triangle $\Phi$ and an adjacent triangle $\Phi^{2}$ as well as the two pairs of generators $\sigma_{1}, \sigma_{2}$ and $\sigma_{1} \sigma_{2}^{2}, \sigma_{2}^{-1}$ of $\Gamma(\mathcal{P})$ associated with them. Moreover, the union of $\Phi$ and $\Phi^{2}$ is a fundamental region for the action of $\Gamma(\mathcal{P})$ on $M$. The map $\mathcal{P}$ on $M$ can be recovered from this action by employing (a combinatorial analogue of) Wythoff's construction, with initial vertex the base vertex in $\Phi$; the latter is fixed by $\sigma_{2}$ (and $\sigma_{2}^{-1}$ ).

Now we can find a model for $\mathcal{P}^{\varphi_{2}}$ as follows. By (2.2), the group of $\mathcal{P}^{\varphi_{2}}$ is generated by $\sigma_{1} \sigma_{2}^{-1}, \sigma_{2}^{2}$, so we can apply Wythoff's construction with the same initial vertex as before. Then the vertices and edges of $\mathcal{P}^{\varphi_{2}}$ are among those of $\mathcal{P}$, and a typical face of $\mathcal{P}^{\varphi_{2}}$ is a hole (2-hole), which is formed by the edge-path which leaves a vertex by the second edge from which it entered, in the same sense (keeping always to the right, say, in some local orientation of $M$ ). Generally, since $q$ is even, only every other edge of $\mathcal{P}$ at a vertex of $\mathcal{P}^{\varphi_{2}}$ belongs to such a face of $\mathcal{P}^{\varphi_{2}}$, so a vertex of $\mathcal{P}^{\varphi_{2}}$ lies in $q / 2$ faces of $\mathcal{P}^{\varphi_{2}}$. The same polyhedron can also be obtained from Wythoff's construction applied with the generators $\sigma_{1} \sigma_{2}^{3}, \sigma_{2}^{-2}$; this pair is the image under $\varphi_{2}$ of the alternative pair $\sigma_{1} \sigma_{2}^{2}, \sigma_{2}^{-1}$ of $\Gamma(\mathcal{P})$.

With appropriate changes, these considerations extend to regular polyhedra $\mathcal{P}($ or $P$ ) and their rotation subgroups $\Gamma^{+}(\mathcal{P})$.

For a survey about realizations of regular or chiral polytopes in euclidean spaces of low dimensions the reader is also referred to [21]. For a discussion of 4-dimensional regular polyhedra see [18].

\section{The Special Group}

We assume from now on that $P$ is a discrete infinite polyhedron, or apeirohedron, which is chiral or regular; in fact, there are no finite chiral polyhedra in $\mathbb{E}^{3}$ (see Section 3 of [24] or see [17]), and the finite regular polyhedra are all known. Then $G=\left\langle S_{1}, S_{2}\right\rangle$ must be an infinite discrete group of isometries. For now we assume that $G$ is (affinely) irreducible on $\mathbb{E}^{3}$, meaning that there is no non-trivial linear subspace $L$ of $\mathbb{E}^{3}$ which is invariant in the sense that $G$ permutes the translates of $L$. It follows that $G$ must be a crystallographic group.

If $R: x \mapsto x R^{\prime}+t$ is a general element of $G$, with $R^{\prime} \in \mathrm{O}_{3}$, the orthogonal group, and $t \in \mathbb{E}^{3}$ is a translation vector, then the linear mappings $R^{\prime}$ form the special group $G_{0}$ of $G$. This is a finite subgroup of $\mathrm{O}_{3}$. (See [10] for the complete enumeration of the finite subgroups of $\mathrm{O}_{3}$.) In particular, if $T(G)$ denotes the subgroup of all translations in $G$, then $G_{0} \cong G / T(G)$. Note that $S_{2}^{\prime}=S_{2}$ and

$$
T^{\prime}=S_{1}^{\prime} S_{2}^{\prime}=S_{1}^{\prime} S_{2} \text {. }
$$


Moreover,

$$
G_{0}=\left\langle S_{1}^{\prime}, S_{2}\right\rangle .
$$

The following refinement of Bieberbach's theorem (see [1] or Section 7.4 of [23]) applies to $G$ and was proved on p. 220 of [20]. (Note that Lemma 7E6 of [20] states the lemma in a slightly weaker form, but its proof actually establishes the stronger version.)

Lemma 3.1. The special group of an irreducible infinite discrete group of isometries in $\mathbb{E}^{2}$ or $\mathbb{E}^{3}$ does not contain rotations of periods other than $2,3,4$ or 6 .

The generators $S_{1}$ and $S_{2}$ of $G$ determine the geometry of the faces and vertex-figures of $P$, respectively. Part I of the paper dealt with the case that $S_{1}$ and $S_{2}$ are rotatory reflections of finite period. When chiral, the corresponding polyhedra in $\mathbb{E}^{3}$ have finite skew faces and finite skew vertex-figures; however, when regular, either the faces or the vertex-figures are planar. Note in this context that an element in $G$ is a rotatory reflection if and only if its image in $G_{0}$ is a rotatory reflection (of the same period). We now investigate the possibility that a generator $S_{1}^{\prime}$ or $S_{2}$ of $G_{0}$ is a rotation of period at least 3 ; the corresponding generator $S_{1}$ or $S_{2}$ of $G$ would then be a rotation or twist (screw motion of infinite period), and the faces or vertex-figures of $P$ would be planar or helical. (Of course, helical vertex-figures are ruled out by our discreteness assumption.)

Before we proceed, note that $S_{1}^{\prime}$ could only be a rotation of period 2 if $P$ had (planar) zigzag faces. We later exclude this possibility (see Lemma 3.7). For now we just observe that the group $G$ of such a polyhedron $P$ must necessarily act reducibly on $\mathbb{E}^{3}$. In fact, $S_{1}$ is necessarily a "half-twist" along the axis of the base (zigzag) face $F_{2}$ (given by a half-turn about this axis, followed by a translation along this axis), and $T$ is either the plane reflection in the perpendicular bisector of the base edge $F_{1}$, or a half-turn about a line through the midpoint of $F_{1}$ and perpendicular to $F_{1}$. In either case, $G_{0}=\left\langle S_{1}^{\prime}, T^{\prime}\right\rangle$ acts reducibly (the axis of $F_{2}$ passes through the midpoint of $F_{1}$ ), and so does $G$.

Moreover, we can also eliminate the possibility that $S_{1}^{\prime}=I$. In fact, in this case $P$ must have linear apeirogons as faces, forcing $P$ to lie entirely on a line (adjacent faces must lie on the same line).

Lemma 3.2. Let $P$ be a discrete chiral or regular apeirohedron in $\mathbb{E}^{3}$ with base vertex $o$, and let the corresponding group $G=\left\langle S_{1}, S_{2}\right\rangle$ act irreducibly, with special group $G_{0}=\left\langle S_{1}^{\prime}, S_{2}\right\rangle$.

(a) The generators $S_{1}$ and $S_{2}$ of $G$ cannot both be rotations.

(b) If one of the generators $S_{1}^{\prime}$ and $S_{2}$ of $G_{0}$ is a rotatory reflection and the other a rotation of period at least 3 , then $G_{0}$ can only be one of the following three groups:

(1) $[3,4]\left(\cong S_{4} \times C_{2}\right)$, the group of a cube $\{4,3\}$ centered at o;

(2) $[3,3]\left(\cong S_{4}\right)$, the subgroup of $[3,4]$ associated with a regular tetrahedron $\{3,3\}$ inscribed in $\{4,3\}$ (as in Fig. 3.1);

(3) the subgroup

$$
[3,3]^{*}:=[3,3]^{+} \cup(-I)[3,3]^{+}\left(\cong A_{4} \times C_{2}\right)
$$

of $[3,4]$, obtained by adjoining $-I$ to the rotation subgroup $[3,3]^{+}$of $\{3,3\}$. 


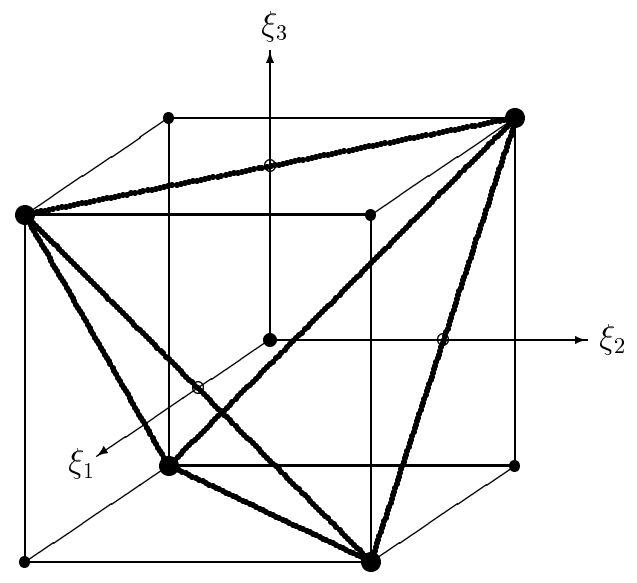

Fig. 3.1. A regular tetrahedron inscribed in a cube.

Proof. Let $P$ be of type $\{p, q\}$; clearly, since $P$ is discrete, $q$ must be finite. Let $p^{\prime}$ denote the period of $S_{1}^{\prime}$. Most values of $p, q$ and $p^{\prime}$ can be ruled out by appealing to Lemma 3.1, or by observing that the group abstractly defined by the relations in (2.1) would be finite.

First we eliminate the possibility that both generators of $G$ are rotations. If $S_{1}$ and $S_{2}$ are rotations, then $G_{0}=\left\langle S_{1}^{\prime}, S_{2}\right\rangle$ must be a finite group in $\mathbb{E}^{3}$ generated by two rotations with distinct axes (recall that $G$ is irreducible). Hence, $G_{0}=[3,3]^{+}$or $[3,4]^{+}$. Since now $p^{\prime}=p$ and every rotation in these groups is of order 3 or 4 , we can only have an infinite group $G$ if $G_{0}=[3,4]^{+}$with $p=q=4$. However, this possibility is also excluded because the product of two rotations of period 4 about distinct axes in $[3,4]^{+}$ cannot be an involution (on the other hand, $T^{\prime}=S_{1}^{\prime} S_{2}$ would have to be an involution). This settles the first part of the lemma.

We now consider the case that $S_{1}^{\prime}$ is a rotation of period at least 3 and that $S_{2}=S_{2}^{\prime}$ is a rotatory reflection. Then $T^{\prime}$ is a plane reflection; note that we cannot have $T^{\prime}=-I$ because $G$ is irreducible. We now apply a similar trick to that in the proof of Theorem $7 \mathrm{E} 4$ of [20] and consider the new mapping

$$
-S_{2}=(-I) S_{2}=-S_{2}^{\prime},
$$

which is a rotation about the same axis as the rotational component of $S_{2}$. Then

$$
\widehat{G}_{0}:=\left\langle S_{1}^{\prime},-S_{2}\right\rangle
$$

is a finite group generated by two rotations with distinct axes, and so we must have $\widehat{G}_{0}=[3,3]^{+}$or $[3,4]^{+}$. Note that $G_{0}$ is a subgroup of $\widehat{G}_{0} \cdot\langle-I\rangle$ and thus of $[3,3]^{*}$ or $[3,4]$, respectively; if $-S_{2}$ is of period 3 , then $-I=S_{2}^{3} \in G_{0}$ and hence $G_{0}=[3,3]^{*}$ or $[3,4]$. Three cases can occur.

If $\widehat{G}_{0}=[3,3]^{+}$, then $G_{0}=[3,3]^{*}$. In this case the elements $S_{1}^{\prime},-S_{2}$ are standard generators of the rotation group $[3,3]^{+}$of $\{3,3\}$, meaning that $S_{1}^{\prime}$ rotates in a face of $\{3,3\}$ and $-S_{2}$ rotates about a vertex of this face, with orientations such that their product 
is a half-turn about the midpoint of an edge of this face containing the vertex. Now $p=3$ or $\infty$, and $q=6$ (already the rotational component of $S_{2}$ is of period 6).

If $\widehat{G}_{0}=[3,4]^{+}$, then $S_{1}^{\prime}$ and $-S_{2}$ must be rotations of period 3 or 4 ; however, they cannot have the same period. In fact, two rotations of period 3 generate a group smaller than $[3,4]^{+}$, and the product of two distinct rotations of period 4 in $[3,4]^{+}$cannot be an involution. Moreover, we can also rule out the case $p=p^{\prime}=3$, because then $G$ would be finite when $q=4$. This leaves the two possibilities that either $\left(p^{\prime}, q\right)=(3,4)$ with $p=\infty$ (the case $p=3$ would again yield a finite group), or $\left(p^{\prime}, q\right)=(4,6)$ with $p=4$ or $\infty$.

In the first case the elements $S_{1}^{\prime},-S_{2}$ are standard generators (in the above sense) of $[3,4]^{+}$, viewed as the rotation group of an octahedron $\{3,4\}$. Now the generators $S_{1}^{\prime}, S_{2}$ of $G_{0}$ are symmetries of a regular tetrahedron inscribed in $\{3,4\}$ (with its vertices at face centers of $\{3,4\})$, so that $G_{0}=[3,3]$.

Similarly, in the second case the elements $S_{1}^{\prime},-S_{2}$ are standard generators of $[3,4]^{+}$, now viewed as the rotation group of the cube $\{4,3\}$. Now $G_{0}=[3,4]$, because $-S_{2}$ has period 3.

Finally, a similar analysis goes through in the dual case when $S_{1}^{\prime}$ is a rotatory reflection (of period $p^{\prime}=p$ ) and $S_{2}$ is a rotation (of period at least 3 ). Now we take

$$
\widehat{G}_{0}:=\left\langle-S_{1}^{\prime}, S_{2}\right\rangle
$$

as the new group.

As before, if $\widehat{G}_{0}=[3,3]^{+}$, then $G_{0}=[3,3]^{*}$ and $-S_{1}^{\prime}, S_{2}$ are standard generators of $[3,3]^{+}$. Now $p=6$ (already the rotational component of $S_{1}$ is of period 6) and $q=3$.

If $\widehat{G}_{0}=[3,4]^{+}$, then again $-S_{1}^{\prime}$ and $S_{2}$ are rotations of distinct periods, 3 or 4 . We can rule out the case $p=4$ (occurring if $-S_{1}^{\prime}$ has period 4), because then $G$ would be finite when $q=3$. This only leaves the possibility that $(p, q)=(6,4)$, obtained when $-S_{1}^{\prime}, S_{2}$ are standard generators of $[3,4]^{+}$, viewed as the rotation group of $\{3,4\}$. Now $G_{0}=[3,4]$, because $-S_{1}^{\prime}$ has period 3 .

In summary, the only possible special groups are [3, 3], [3, 4] and [3, 3]*, with generators $S_{1}^{\prime}, S_{2}$ specified as above.

The next two lemmas employ the geometry of $P$ to eliminate the possibility of finite faces in Lemma 3.2(b). In each case we exhibit representations of the putative generators $S_{1}, S_{2}$ of $G$ and prove that these generators must actually determine a regular polyhedron which either has helical faces or is finite; however, the latter possibility is excluded by our assumptions on $P$. The results are interesting in their own right.

Lemma 3.3. Let $P$ be a discrete chiral or regular apeirohedron in $\mathbb{E}^{3}$ with base vertex $o$, and let the corresponding group $G=\left\langle S_{1}, S_{2}\right\rangle$ be irreducible, with special group $G_{0}=\left\langle S_{1}^{\prime}, S_{2}\right\rangle$. If $S_{1}^{\prime}$ is a rotation of period at least 3 and $S_{2}$ a rotatory reflection, then $P$ is a regular polyhedron with helical faces and $S_{1}$ is a twist. In particular, $P$ is the Petrial of a Petrie-Coxeter polyhedron $\{4,6 \mid 4\},\{6,4 \mid 4\}$ or $\{6,6 \mid 3\}$; that is,

$$
P=\{\infty, 6\}_{4,4},\{\infty, 4\}_{6,4} \quad \text { or } \quad\{\infty, 6\}_{6,3},
$$

respectively (notation as on p. 224 of [20]). 
Proof. By Lemma 3.2, $G_{0}$ must be one of the groups [3, 3] $]^{*}$ [3, 3] or [3, 4]. In each case $G_{0}$ is associated with standard generators of $\widehat{G}_{0}:=\left\langle S_{1}^{\prime},-S_{2}\right\rangle$. We now investigate the possible generators $S_{1}, S_{2}$ of $G$.

We begin with the group $[3,3]^{*}$. Now $S_{1}^{\prime},-S_{2}$ are standard generators of $[3,3]^{+}$. Once $S_{2}$ has been selected, there are three admissible choices for $S_{1}^{\prime}$ such that $S_{1}^{\prime} S_{2}$ has period 2; if $S_{1}^{\prime}$ is one of them, then the two others are $S_{2}^{-1} S_{1}^{\prime} S_{2}$ and $S_{2}^{-2} S_{1}^{\prime} S_{2}^{2}$. This follows directly from Lemma 5.1 of [24], applied to the rotatory reflections $S_{2}$ and $-S_{1}^{\prime}$. There is also the further possibility of reversing the orientation of the generator $S_{2}$ and replacing it by its inverse $S_{2}^{-1}$. However, this simply amounts to replacing the polyhedron $P$ by its enantiomorphic image; the latter is the same underlying polyhedron, with the same group, but with the new distinguished generators $S_{1} S_{2}^{2}, S_{2}^{-1}$ (determined by a new, adjacent base flag); see Section 2 for more details.

Thus, without loss of generality we may assume that

$$
\begin{aligned}
& S_{1}^{\prime}: x \mapsto\left(\xi_{2},-\xi_{3},-\xi_{1}\right), \\
& S_{2}: \quad x \mapsto-\left(\xi_{3}, \xi_{1}, \xi_{2}\right),
\end{aligned}
$$

described in terms of $x=\left(\xi_{1}, \xi_{2}, \xi_{3}\right)$, so that $T^{\prime}=S_{1}^{\prime} S_{2}$ is the reflection in the $\xi_{1} \xi_{3}$ plane. (Note that $-S_{1}^{\prime}, S_{2}$ are the generators for the special group of the group $G(a, b)$ in (5.1) of [24].) Then $T=S_{1} S_{2}$ must be a reflection in a plane parallel to the $\xi_{1} \xi_{3}$-plane, and since $T$ interchanges the vertices in the base edge $F_{1}$, this plane must coincide with the perpendicular bisector of $F_{1}$. In particular, the vertex $v:=o T$ of $F_{1}$ distinct from $o$ must be of the form $v=(0, a, 0)$ for some non-zero parameter $a$. It follows that $G$ is generated by

$$
\begin{aligned}
& S_{2}: \quad x \mapsto-\left(\xi_{3}, \xi_{1}, \xi_{2}\right), \\
& T: \quad x \mapsto\left(\xi_{1},-\xi_{2}, \xi_{3}\right)+(0, a, 0) .
\end{aligned}
$$

Since $S_{1}=T S_{2}^{-1}$, we then have

$$
S_{1}: \quad x \mapsto\left(\xi_{2},-\xi_{3},-\xi_{1}\right)+(-a, 0,0),
$$

so, in particular,

$$
S_{1}^{3}: \quad x \mapsto\left(\xi_{1}, \xi_{2}, \xi_{3}\right)+a(-1,-1,1),
$$

which is a non-trivial translation. Hence, $S_{1}$ must be a twist and $P$ must have helical faces (spiraling over an equilateral triangle). Note that $P$ is of type $\{\infty, 6\}$.

We now employ Lemma 2.1 to show that $P$ must be regular. It is straightforward to check that the reflection $R$ (in the plane $\xi_{1}=\xi_{3}$ ) given by

$$
R: \quad x \mapsto\left(\xi_{3}, \xi_{2}, \xi_{1}\right)
$$

satisfies the conditions required in Lemma 2.1 with

$$
F_{0}=o, \quad F_{1}=\{o, v\}, \quad F_{2}=o\left\langle S_{1}\right\rangle, \quad F_{2}^{\prime}=F_{2} T .
$$

In fact, observe that conjugation by $R$ transforms the pair $S_{1}, S_{2}$ to the new generating pair $S_{1} S_{2}^{2}, S_{2}^{-1}$, so in particular $F_{2}=o\left\langle S_{1}\right\rangle$ is mapped to $F_{2}^{\prime}=o\left\langle S_{1} S_{2}^{2}\right\rangle$. Hence, $P$ is 
regular and $R=R_{2}$. Inspection of the list of 12 regular apeirohedra (with an irreducible group) shows that $P=\{\infty, 6\}_{4,4}$ (see p. 224 of [20]), the Petrial of the Petrie-Coxeter polyhedron $\{4,6 \mid 4\}$ (see [5]), with Petrie polygons of length 4 (indicated by the first subscript in the symbol for $P$ ); note here that $S_{1}^{2} S_{2}^{2}\left(=\left(R_{0} R_{1} R_{2}\right)^{2}\right)$ has period 2.

Next we investigate the possible generators $S_{1}, S_{2}$ of $G$ when $G_{0}=[3,4]$. Now $S_{1}^{\prime},-S_{2}$ are standard generators of $[3,4]^{+}$, viewed as the rotation group of a cube $\{4,3\}$. As in the previous case, once $S_{2}$ has been selected, there are precisely three admissible choices for $S_{1}^{\prime}$ such that $S_{1}^{\prime} S_{2}$ has period 2; if $S_{1}^{\prime}$ is one of them, then the two others are $S_{2}^{-1} S_{1}^{\prime} S_{2}$ and $S_{2}^{-2} S_{1}^{\prime} S_{2}^{2}$. This follows from Lemma 6.1 of [24], applied to the rotatory reflections $S_{2}$ and $-S_{1}^{\prime}$. Moreover, the same remark as above about replacing $S_{2}$ by $S_{2}^{-1}$ applies in this context.

Thus we may take as generators

$$
\begin{array}{llll}
S_{1}^{\prime}: & x & \mapsto & \left(\xi_{1},-\xi_{3}, \xi_{2}\right), \\
S_{2}: & x & \mapsto & -\left(\xi_{3}, \xi_{1}, \xi_{2}\right),
\end{array}
$$

so that $T^{\prime}$ is the reflection in the plane $\xi_{1}+\xi_{2}=0$. (Now $-S_{1}^{\prime}, S_{2}$ are the generators for the special group of the group $H(c, d)$ in (6.1) of [24].) The mirror of $T$ once again is the perpendicular bisector of $F_{1}$ and is parallel to the mirror of $T^{\prime}$. Hence, if $v:=o T$, then $v=(a, a, 0)$ for some non-zero parameter $a$. It follows that $G$ is generated by

$$
\begin{aligned}
& S_{2}: x \mapsto-\left(\xi_{3}, \xi_{1}, \xi_{2}\right), \\
& T: \quad x \mapsto\left(-\xi_{2},-\xi_{1}, \xi_{3}\right)+(a, a, 0) .
\end{aligned}
$$

The element $S_{1}=T S_{2}^{-1}$ is given by

$$
S_{1}: x \mapsto\left(\xi_{1},-\xi_{3}, \xi_{2}\right)+(-a, 0,-a),
$$

so, in particular,

$$
S_{1}^{4}: \quad x \quad \mapsto \quad\left(\xi_{1}, \xi_{2}, \xi_{3}\right)+a(-4,0,0),
$$

which is again a non-trivial translation. Hence, $S_{1}$ must be a twist and $P$ must have helical faces (spiraling over a square).

As in the previous case we can prove that $P$ is regular. The element $R\left(=R_{2}\right)$ is the reflection (in the plane $\xi_{1}=\xi_{2}$ ) given by

$$
R: \quad x \mapsto\left(\xi_{2}, \xi_{1}, \xi_{3}\right) .
$$

In this case, $P=\{\infty, 6\}_{6,3}$, the Petrial of the Petrie-Coxeter polyhedron $\{6,6 \mid 3\}$, with Petrie polygons of length 6 (again indicated by the first subscript in the symbol for $P$ ); now $S_{1}^{2} S_{2}^{2}$ has period 3 .

Finally, when $G_{0}=[3,3]\left(\cong S_{4}\right)$, we must take a rotation $S_{1}^{\prime}$ of period 3 and a rotatory reflection $S_{2}$ of period 4 as generators of $G_{0}$. Once $S_{2}$ has been selected, there are now exactly four admissible choices for $S_{1}^{\prime}$; if $S_{1}^{\prime}$ is one of them, then the three others are $S_{2}^{-j} S_{1}^{\prime} S_{2}^{j}$ for $j=1,2,3$. Moreover, the same remark as before about replacing $S_{2}$ by $S_{2}^{-1}$ applies.

Thus, with

$$
\begin{aligned}
& S_{1}^{\prime}: x \mapsto\left(\xi_{2}, \xi_{3}, \xi_{1}\right), \\
& S_{2}: \quad x \mapsto\left(-\xi_{1},-\xi_{3}, \xi_{2}\right),
\end{aligned}
$$


we obtain the same $v, T^{\prime}$ and $T$ as in the previous case. In particular, we have

$$
\begin{aligned}
& S_{1}: x \mapsto\left(\xi_{2}, \xi_{3}, \xi_{1}\right)+(-a, 0,-a), \\
& S_{1}^{3}: x \mapsto\left(\xi_{1}, \xi_{2}, \xi_{3}\right)+a(-2,-2,-2) .
\end{aligned}
$$

Once again, $P$ is regular, by Lemma 2.1. Now $R\left(=R_{2}\right)$ is given by the reflection (in the plane $\xi_{3}=0$ )

$$
R: \quad x \mapsto\left(\xi_{1}, \xi_{2},-\xi_{3}\right),
$$

and $P=\{\infty, 4\}_{6,4}$, the Petrial of $\{6,4 \mid 4\}$, with dimension vector $(1,1,2)$ (see p. 224 of [20]).

The three polyhedra occurring in Lemma 3.3 are precisely the pure regular polyhedra with dimension vector $(1,1,2)$ (see p. 225 of [20]). Note that the values $1,1,2$ for the dimensions of the mirrors for their generating reflections $R_{0}, R_{1}, R_{2}$ accord with the geometric type of the generators of $G$, namely a twist for $S_{1}\left(=R_{0} R_{1}\right)$ and a rotatory reflection for $S_{2}\left(=R_{1} R_{2}\right)$.

Lemma 3.4. Let $P$ be a discrete chiral or regular apeirohedron in $\mathbb{E}^{3}$, and let the corresponding group $G=\left\langle S_{1}, S_{2}\right\rangle$ be irreducible. Then the generators cannot be such that $S_{1}$ is a rotatory reflection and $S_{2}$ a rotation.

Proof. Suppose that $S_{1}$ (and hence $S_{1}^{\prime}$ ) is a rotatory reflection and $S_{2}$ a rotation. Then $G_{0}=[3,3]^{*}$ or $[3,4]$, by the proof of Lemma 3.2. We treat these cases separately and appeal to the discussion of the groups occurring in the proof of Lemma 3.3. Again, $o$ is the base vertex.

If $G_{0}=[3,3]^{*}$, then we may take

$$
\begin{aligned}
& S_{1}^{\prime}: \quad x \mapsto-\left(\xi_{2}, \xi_{3}, \xi_{1}\right), \\
& S_{2}: x \mapsto\left(-\xi_{3}, \xi_{1},-\xi_{2}\right),
\end{aligned}
$$

which are the inverses of the generators $S_{2}$ and $S_{1}^{\prime}$ of (3.1), respectively, of periods 6 and 3. Then $T^{\prime}, T$ and $v$ of (3.1) remain unchanged, because $T$ and $T^{\prime}$ have period 2. In particular, $G$ is generated by

$$
\begin{aligned}
& S_{2}: x \mapsto\left(-\xi_{3}, \xi_{1},-\xi_{2}\right), \\
& T: \quad x \mapsto\left(\xi_{1},-\xi_{2}, \xi_{3}\right)+(0, a, 0),
\end{aligned}
$$

and $S_{1}=T S_{2}^{-1}$ is given by

$$
S_{1}: x \mapsto\left(-\xi_{2},-\xi_{3},-\xi_{1}\right)+(a, 0,0) .
$$

However, then the whole group $G$ fixes the point $z:=\frac{1}{2}(a, a,-a)$, and so $G$ must be a finite group, contrary to our assumption that $P$ is infinite. (This finite group consists of the symmetries of the cube of edge length $a$ centered at $z$, and the associated polyhedron is the Petrial $\{6,3\}_{4}$ of $\{4,3\}$; see pp. 192 and 218 of [20].) 
In a similar fashion we deal with the case $G_{0}=[3,4]$. We take

$$
\begin{array}{llll}
S_{1}^{\prime}: & x & \mapsto & -\left(\xi_{2}, \xi_{3}, \xi_{1}\right), \\
S_{2}: & x & \mapsto & \left(\xi_{1}, \xi_{3},-\xi_{2}\right),
\end{array}
$$

which are the inverses of the generators $S_{2}$ and $S_{1}^{\prime}$ of (3.6), respectively, of periods 6 and 4. Then $T^{\prime}, T$ and $v$ of (3.6) remain unchanged and $G$ is generated by any two of

$$
\begin{aligned}
& S_{1}: \quad x \quad \mapsto \quad-\left(\xi_{2}, \xi_{3}, \xi_{1}\right)+(a, 0, a), \\
& S_{2}: x \mapsto\left(\xi_{1}, \xi_{3},-\xi_{2}\right) \text {, } \\
& T: \quad x \mapsto\left(-\xi_{2},-\xi_{1}, \xi_{3}\right)+(a, a, 0) .
\end{aligned}
$$

Then $G$ fixes $z^{\prime}:=(a, 0,0)$, so it must again be a finite group, contrary to our assumptions on $P$. (Now this finite group consists of the symmetries of the octahedron with vertices $z^{\prime} \pm a e_{1}, z^{\prime} \pm a e_{2}, z^{\prime} \pm a e_{3}$, where $e_{1}, e_{2}, e_{3}$ is the standard basis of $\mathbb{E}^{3}$; the associated polyhedron is $\{6,4\}_{3}$, the Petrial of $\{3,4\}$.)

The above analysis shows that, for a discrete chiral apeirohedron $P$ with an irreducible group $G$, either the generators $S_{1}, S_{2}$ of $G$ must be rotatory reflections, or $S_{1}$ must be a twist and $S_{2}$ a rotation.

If $P$ has finite faces, then both generators must be rotatory reflections of finite period, and $P$ must be among the polyhedra enumerated in Part I [24]. In particular, this completes the proof of the following theorem, at least in the irreducible case; the reducible case is settled by Lemma 3.7.

Theorem 3.5. There are no discrete chiral polyhedra with finite faces in $\mathbb{E}^{3}$ other than those (with skew faces and skew vertex-figures) enumerated in Part I.

For a chiral apeirohedron $P$ with infinite, helical faces, $S_{1}$ must be a twist and $S_{2}$ a rotation. In particular, $G_{0}$ is generated by the rotations $S_{1}^{\prime}, S_{2}$ (with distinct axes), so $G_{0}$ must be a finite group of rotations in $\mathbb{E}^{3}$. Bearing in mind that $S_{1}^{\prime} S_{2}$ must have period 2, we immediately arrive at

Lemma 3.6. Let $P$ be a discrete chiral or regular apeirohedron in $\mathbb{E}^{3}$ with helical faces, and let o be its base vertex. Let the corresponding group $G=\left\langle S_{1}, S_{2}\right\rangle$ act irreducibly, with special group $G_{0}=\left\langle S_{1}^{\prime}, S_{2}\right\rangle$, and let $S_{2}$ be a rotation. Then $G_{0}=[3,3]^{+}$or $[3,4]^{+}$. Moreover, if $p^{\prime}$ denotes the period of $S_{1}^{\prime}$ and $P$ is of type $\{\infty, q\}$, then $\left(p^{\prime}, q\right)=(3,3)$, $(3,4)$ or $(4,3)$, and the faces of $P$ are helices over equilateral triangles or squares according as $p^{\prime}=3$ or 4 .

In the next three sections we enumerate the discrete chiral apeirohedra with helical faces. They occur in three real-valued two-parameter families, each of type $\{\infty, 3\}$ or $\{\infty, 4\}$. In each case, $T=S_{1} S_{2}$ is a half-turn whose rotation axis is perpendicular to the base edge of $P$; in particular, this accounts for the two independent parameters describing the base vertex $v=o T$. By contrast, observe that, in the situations discussed in Lemmas 3.3 and 3.4, the element $T$ is a plane reflection, so that we have only one 
independent parameter; this accords with $P$ being regular in these cases, and being determined up to similarity.

Finally, then, we must settle the reducible case. Recall that there are 12 non-planar regular polyhedra in $\mathbb{E}^{3}$ with a reducible group, namely the blended polyhedra

$$
\{p, q\} \#\{\}, \quad\{p, q\} \#\{\infty\}, \quad\{p, q\}^{\pi} \#\{\}, \quad\{p, q\}^{\pi} \#\{\infty\},
$$

where the first component is either a regular tessellation $\{p, q\}=\{4,4\},\{3,6\}$ or $\{6,3\}$, or the Petrial $\{p, q\}^{\pi}$ of $\{p, q\}$, and where the second component is either a segment \{\} or an apeirogon $\{\infty\}$ (see pp. 221-223 of [20]). The following lemma says that reducible groups can be eliminated from further discussion.

Lemma 3.7. A discrete chiral apeirohedron in $\mathbb{E}^{3}$ must have an irreducible symmetry group.

Proof. It is not possible for a discrete chiral polyhedron in $\mathbb{E}^{3}$ to be planar (see Theorem 3.2 of [24] or see [17]), so it suffices to consider non-planar polyhedra. Let $P$ be a discrete, non-planar polyhedron, and let $P$ be chiral or regular. Suppose the corresponding group $G=\left\langle S_{1}, S_{2}\right\rangle$ is reducible. We show that $P$ is regular.

Since $G$ is reducible, there exists a plane $L$ through $o$ such that $G$ permutes the translates of $L$ as well as the translates of $L^{\perp}$, the orthogonal complement of $L$ at $o$. In particular, since $o S_{2}=o$, the subspaces $L$ and $L^{\perp}$ are invariant under $S_{2}$, and $S_{2}$ is either a rotation (about $L^{\perp}$ ) or a rotatory reflection (with a rotation about $L^{\perp}$ as its rotation component). Moreover, the vertex $v$ of the base edge $F_{1}$ distinct from $o$ cannot lie in $L$; otherwise, the vertex-figure at $o$, and hence at every vertex, would lie in $L$, making $P$ planar. We shall see that the geometric type of $S_{2}$ determines the second component of the blend.

Now suppose $S_{2}$ is a rotation. Let $L^{\prime}$ denote the plane parallel to $L$ through $v$. Then $P$ has all its vertices in $L$ or $L^{\prime}$, and the vertex-figure at a vertex in one plane is a flat (finite or infinite) polygon contained in the other plane; in particular, the edge-graph of $P$ is bipartite.

If the faces of $P$ are finite (skew) polygons, then the generator $S_{1}$ is a rotatory reflection, so $T$ can only be the point reflection in $\frac{1}{2} v$; in particular, $S_{1}^{\prime}=T^{\prime} S_{2}^{\prime-1}=$ $(-I) S_{2}^{\prime-1}$. The projection of $P$ on $L$ is discrete, and since $S_{2}$ and the rotation component of $S_{1}^{\prime}$ must be rotations of periods 2, 3, 4 or 6, it is not hard to see (using basic properties of Wythoff's construction) that it must be a tessellation $\{p, q\}$; hence $P=\{p, q\} \#\{\}$.

Similarly, if the faces are infinite (zigzags), then $S_{1}$ is a half-twist about a line parallel to $L$ (a half-turn about the axis of the base face, followed by a translation along this axis), so $T$ must be a half-turn. Now the projection on $L$ is the Petrial $\{p, q\}^{\pi}$, and $P=\{p, q\}^{\pi} \#\{\}$.

Now let $S_{2}$ be a rotatory reflection. Let $L_{k}$ denote the plane parallel to $L$ through the point $k v$, for $k \in \mathbb{Z}$. Now the vertices adjacent to $o\left(\in L_{0}\right)$ alternate between $L_{-1}$ and $L_{1}$. It follows that $P$ has all its vertices in the union of the planes $L_{k}$, and that the vertices adjacent to a vertex in one of the planes alternate between the adjacent planes. Hence the faces are helices or zigzags, each with one vertex on each plane $L_{k}$.

If the faces of $P$ are helices, then $S_{1}$ is a twist about an axis (of the base face) parallel to $L^{\perp}$, which is determined by a rotation of finite period $p^{\prime}$ (say) in $L$ and a translation 
along this axis. The projection of $P$ on $L$ is again discrete. This can be seen as follows. Let $D$ be a circular disc in $L$ centered at $o$, and let $\widehat{D}$ be a right cylinder over $D$ with height $|t|$, where $t$ is the translation vector of the translation $S_{1}^{p^{\prime}}$. Now, if $x$ is a vertex of $P$ projecting on $D$, then $\widehat{x}:=x+n t=x S_{1}^{n p^{\prime}}$, for some $n \in \mathbb{Z}$, is a vertex of $P$ in $\widehat{D}$ which projects on the same point as $x$. Thus the vertices in $\widehat{D}$ represent all the vertices projecting on $D$. However, since $P$ is discrete, it only has finitely many vertices in $\widehat{D}$, so its projection only has finitely many points in $D$. This proves that the projection of $P$ on $L$ is discrete. As before we then establish that this projection is a tessellation $\{p, q\}$ in $L$. Hence $P=\{p, q\} \#\{\infty\}$.

Finally, if the faces of $P$ are zigzags, then $S_{1}$ must be a glide reflection whose respective reflection mirror is perpendicular to $L$ and contains the axis of the base face. Moreover, $T$ is a half-turn about a line parallel to $L$. The projection of $P$ on $L$ is again discrete, for similar reasons as in the previous case (now $S_{1}^{2}$ is a translation). Now this projection is a Petrial $\{p, q\}^{\pi}$, and $P=\{p, q\}^{\pi} \#\{\infty\}$. This concludes the proof.

\section{Type $\{\infty, 3\}$, with Helical Faces over Triangles}

In this section we enumerate the chiral apeirohedra of type $\{\infty, 3\}$ whose faces are helices over triangles. They occur when $G_{0}=[3,3]^{+}$in Lemma 3.6. Throughout we may assume that the symmetry group $G$ acts irreducibly; we saw in Lemma 3.7 that the reducible case adds no further polyhedra to the list.

Each polyhedron is obtained from a suitable group $G$ by Wythoff's construction with initial (or base) vertex $F_{0}:=o$. For a polyhedron $P$ of type $\{\infty, 3\}$ with helical faces over triangles, we must necessarily begin with a group $G=\left\langle S_{1}, S_{2}\right\rangle$ whose special group is $G_{0}=[3,3]^{+}$. Then $S_{1}^{\prime}, S_{2}$ are standard generators of $[3,3]^{+}$, and each is a rotation of period 3. As in similar situations discussed earlier, once $S_{2}$ has been selected, there are three equivalent choices for $S_{1}^{\prime}$ such that $S_{1}^{\prime} S_{2}$ has period 2; if $S_{1}^{\prime}$ is one of them, then the other two are $S_{2}^{-1} S_{1}^{\prime} S_{2}$ and $S_{2}^{-2} S_{1}^{\prime} S_{2}^{2}$. Moreover, as mentioned before, if we substitute $S_{2}$ by its inverse, we arrive at (the enantiomorphic image of) the same underlying polyhedron, so we need not investigate this case separately.

Thus we may take

$$
\begin{aligned}
& S_{1}^{\prime}: x \mapsto\left(-\xi_{3},-\xi_{1}, \xi_{2}\right), \\
& S_{2}: \quad x \mapsto\left(\xi_{2}, \xi_{3}, \xi_{1}\right) .
\end{aligned}
$$

Then $T^{\prime}=S_{1}^{\prime} S_{2}$ is the half-turn about the $\xi_{2}$-axis. Since $T=S_{1} S_{2}$ must be a half-turn interchanging the two vertices in the base edge $F_{1}$, its rotation axis must necessarily be perpendicular to $F_{1}$ and pass through the midpoint of $F_{1}$. However, this axis is parallel to the $\xi_{2}$-axis, so $F_{1}$ must lie in the $\xi_{1} \xi_{3}$-plane. Hence, $v:=o T=(a, 0, b)$ for some real parameters $a$ and $b$, not both zero, so that we have $G=G_{1}(a, b)$, the group generated by

$$
\begin{aligned}
S_{2}: \quad x & \mapsto\left(\xi_{2}, \xi_{3}, \xi_{1}\right), \\
T: \quad x & \mapsto\left(-\xi_{1}, \xi_{2},-\xi_{3}\right)+(a, 0, b) .
\end{aligned}
$$


From $S_{1}=T S_{2}^{-1}$ we then obtain

$$
\begin{aligned}
& S_{1}: x \mapsto\left(-\xi_{3},-\xi_{1}, \xi_{2}\right)+(b, a, 0), \\
& S_{1}^{3}: \quad x \mapsto\left(\xi_{1}, \xi_{2}, \xi_{3}\right)+(b-a)(1,-1,-1) \text {. }
\end{aligned}
$$

In particular, $S_{1}^{3}$ is the translation by $(b-a)(1,-1,-1)$, and is trivial if $b=a$. Hence the case $b=a$ is special and yields the finite group

$$
G_{1}(a, a) \cong[3,3]^{+}
$$

(with fixed point $\frac{1}{2}(a, a, a)$ ); the corresponding polyhedron is a regular tetrahedron. It is often convenient to exclude this case from the discussion.

When necessary, we indicate the parameters $a$ and $b$ more explicitly and write $T=$ $T(a, b), S_{1}=S_{1}(a, b)$ and so on; the generator $S_{2}$ does not actually depend on $a, b$.

The polyhedron $P=P_{1}(a, b)$ is obtained from $G=G_{1}(a, b)$ by Wythoff's construction with base vertex $F_{0}=o$. The base edge $F_{1}$ has vertices $o$ and $v$. If we identify faces of $P$ with their vertex-sets, then a helical face $F$ (over a triangle) is denoted as follows: if $x, y, z$ are consecutive vertices of $F$ and $t$ is the translation vector associated with $F$, then

$$
F=\{x, y, z\}+\mathbb{Z} \cdot t,
$$

with the understanding that the order in which the vertices of $F$ occur is given by

$\ldots, x-2 t, y-2 t, z-2 t, x-t, y-t, z-t, x, y, z, x+t, y+t, z+t, x+2 t, y+2 t, z+2 t, \ldots$.

The same notation, with $t=o$, is also adopted in the special case $b=a$; then $F=$ $\{x, y, z\}$, so we usually drop the term $\mathbb{Z} \cdot t$. In particular, for the base face we have

$$
F_{2}=o\left\langle S_{1}\right\rangle=\{(a, 0, b),(0,0,0),(b, a, 0)\}+\mathbb{Z} \cdot c(1,-1,-1),
$$

where $c:=b-a$. The remaining vertices, edges and faces of $P$ are the images of $F_{0}$, $F_{1}$ and $F_{2}$ under $G$. The vertices adjacent to $o$ are

$$
v=(a, 0, b), \quad v S_{2}=(0, b, a), \quad v S_{2}^{2}=(b, a, 0) .
$$

If the polyhedra $P_{1}(a, b)$ are only considered up to similarity, then the two parameters $a, b$ can be reduced to a single parameter. This is similar to what we observed for the polyhedra in [24]. In fact, if $s$ is a non-zero scalar and $R_{s}:=s I$, then conjugation of the generators $S_{2}, T(a, b)$ for $G_{1}(a, b)$ by $R_{s}$ gives the generators $S_{2}, T(s a, s b)$ for $G_{1}(s a, s b)$. It follows that

$$
P_{1}(s a, s b)=P_{1}(a, b) R_{s}=: s P_{1}(a, b),
$$

so $P_{1}(s a, s b)$ and $P_{1}(a, b)$ are similar. In particular, $P_{1}(a, b)$ is similar to $P_{1}(1, b / a)$ or $P_{1}(0,1)$ according as $a \neq 0$ or $a=0$.

Before we move on, observe that the polyhedra $P_{1}(a, b)$ of type $\{\infty, 3\}$ are related to the chiral (or regular) polyhedra $P(a, b)$ of type $\{6,6\}$ described in Section 5 of [24] (their notation has no suffix). These polyhedra have finite skew hexagonal faces and vertex-figures. The relationship is established by the facetting operation $\varphi_{2}$ defined in 
(2.2) (or, equivalently, by the operation $\kappa$ discussed in Section 8). Recall from (5.1) and (5.2) of [24] that the generators $\widehat{S}_{1}, \widehat{S}_{2}$ (say) and the corresponding element $\widehat{T}:=\widehat{S}_{1} \widehat{S}_{2}$ of the symmetry group $G(a, b)$ of $P(a, b)$ are given by

$$
\begin{aligned}
& \widehat{S}_{1}: \quad x \mapsto\left(-\xi_{2}, \xi_{3}, \xi_{1}\right)+(0,-b,-a), \\
& \widehat{S}_{2}: x \mapsto-\left(\xi_{3}, \xi_{1}, \xi_{2}\right) \text {, } \\
& \widehat{T}: \quad x \mapsto\left(-\xi_{1}, \xi_{2},-\xi_{3}\right)+(a, 0, b) .
\end{aligned}
$$

We now have the following:

Lemma 4.1. $\quad P_{1}(a, b)=P(a, b)^{\varphi_{2}}$, for all real parameters $a$ and $b$.

Proof. In the present context, $\varphi_{2}$ takes the form

$$
\varphi_{2}: \quad\left(\widehat{S}_{1}, \widehat{S}_{2}\right) \mapsto\left(\widehat{S}_{1} \widehat{S}_{2}^{-1}, \widehat{S}_{2}^{2}\right) .
$$

Now observe that, when $\varphi_{2}$ is expressed in terms of the generators $\widehat{S}_{2}, \widehat{T}$ of $G(a, b)$, we obtain

$$
\varphi_{2}: \quad\left(\widehat{S}_{2}, \widehat{T}\right) \mapsto\left(\widehat{S}_{2}^{2}, \widehat{T}\right)=\left(S_{2}, T\right) .
$$

Hence $P_{1}(a, b)=P(a, b)^{\varphi_{2}}$ and $G_{1}(a, b)$ is a subgroup of $G(a, b)$.

Note that the above relationship between $P(a, b)$ and $P_{1}(a, b)$ holds without any restriction on the parameters. On the other hand, $P(a, b)$ is known to be discrete only when $a$ and $b$ can be scaled to a pair of relatively prime integers, whereas there is no such condition for $P_{1}(a, b)$. Thus we may (in most cases, do) have the interesting phenomenon that the derived polyhedron $P_{1}(a, b)$ is discrete but the original polyhedron $P(a, b)$ is non-discrete. We can conclude from this that then there are infinitely many copies of $P_{1}(a, b)$ inscribed in $P(a, b)$.

We already note here two special cases. If $b=-a$, then the polyhedra are similar to those obtained for $(a, b)=(1,-1)$, and are regular. In particular, since $P(1,-1)=$ $\{6,6\}_{4}$ (see Theorem 5.17 of [24]) and $\{6,6\}_{4}^{\varphi_{2}}=\{\infty, 3\}^{(a)}$ (notation as on p. 224 of [20]), we observe that

$$
P_{1}(1,-1)=\{\infty, 3\}^{(a)} .
$$

Similarly, the case $b=a$ reduces to $(a, b)=(1,1)$ and yields regular polyhedra. Now $P(1,1)=\{6,6 \mid 3\}$ and $\{6,6 \mid 3\}^{\varphi_{2}}=\{3,3\}$ (again, see Theorem 5.17 of [24] and p. 224 of [20]), so, in particular,

$$
P_{1}(1,1)=\{3,3\} .
$$

It follows from our discussion in Section 2 that the faces of the polyhedron $P_{1}(a, b)$ are among the holes of $P(a, b)$. These holes are helices over triangles, or triangles, according as $b \neq a$ or $b=a$.

We now determine the translation subgroup $T\left(G_{1}(a, b)\right)$ of $G_{1}(a, b)$. When convenient, we identify a translation with its translation vector. It is convenient to use the following notation for lattices. Let $s$ be a non-zero real number, let $k=1,2$ or 3 and 
let $\mathbf{s}:=\left(s^{k}, 0^{3-k}\right)$, the vector with $k$ components $s$ and $3-k$ components 0 . Following p. 166 of [20], we write $\Lambda_{s}$ for the sublattice of the cubic lattice $\mathbb{Z}^{3}$ generated by $\mathbf{s}$ and its images under permutation and changes of sign of coordinates. Then

$$
\Lambda_{\mathbf{s}}=s \Lambda_{\left(1^{k}, 0^{3-k}\right)} .
$$

Note that $\Lambda_{(1,0,0)}=\mathbb{Z}^{3}$. The lattice $\Lambda_{(1,1,0)}$ is the face-centered cubic lattice (the root lattice $D_{3}$ ) consisting of all integral vectors whose coordinate sum is even, and $\Lambda_{(1,1,1)}$ is the body-centered cubic lattice (see [4]).

We know from (4.3) that $S_{1}^{3}$ is the translation by $c(1,-1,-1)$, where again $c:=b-a$. Hence, if $R$ is any element of $G=G_{1}(a, b)$ and $R^{\prime}$ is its image in the special group $G_{0}$, then $R^{-1} S_{1}^{3} R$ is the translation by $c(1,-1,-1) R^{\prime}$. Since $G_{0} \cong[3,3]^{+}$, we then have translations by the vectors $c(1,1,1), c(1,-1,-1), c(-1,1,-1), c(-1,-1,1)$ in $G$, and hence also by their integral linear combinations. It follows that

$$
c \Lambda_{(1,1,1)} \leqslant T(G) .
$$

(The left-hand side is trivial if $b=a$.) In fact, we have the following stronger result.

Lemma 4.2. The translation subgroup of $G_{1}(a, b)$ is given by $T\left(G_{1}(a, b)\right)=c \Lambda_{(1,1,1)}$, with $c=b-a$.

Proof. Suppose $b \neq a$. Since $G=G_{1}(a, b)=\left\langle S_{2}, T\right\rangle$, we have $G=N \cdot\left\langle S_{2}\right\rangle$ (as a product of subgroups), where $N:=\left\langle T_{1}, T_{2}, T_{3}\right\rangle$ with $T_{2}:=T, T_{1}:=S_{2}^{-1} T S_{2}$ and $T_{3}:=S_{2}^{-2} T S_{2}^{2}$. Bearing in mind that the generator $S_{2}$ for $P_{1}(a, b)$ is just the square of the generator $\widehat{S}_{2}$ for $P(a, b)$ (see (4.8)), we see that $T_{1}, T_{2}, T_{3}$ are the half-turns occurring in (5.1) and (5.6) of [24] with the same notation, namely

$$
\begin{aligned}
& T_{1}: \quad x \mapsto\left(\xi_{1},-\xi_{2},-\xi_{3}\right)+(0, b, a), \\
& T_{2}: x \mapsto\left(-\xi_{1}, \xi_{2},-\xi_{3}\right)+(a, 0, b), \\
& T_{3}: \quad x \mapsto\left(-\xi_{1},-\xi_{2}, \xi_{3}\right)+(b, a, 0) .
\end{aligned}
$$

Observe that a translation in $G$ must necessarily belong to $N$; in fact, in the special group, the images of a translation in $G$ or of an element in $N$ must involve an even number of sign changes but no permutation of coordinates, whereas a non-trivial element in $\left\langle S_{2}\right\rangle$ involves a non-trivial permutation of coordinates.

It is straightforward to check that each product $T_{i} T_{j} T_{k}$, with $i, j, k$ distinct, is a translation in $G$. The translation vectors of $T_{1} T_{3} T_{2}, T_{2} T_{1} T_{3}$ and $T_{3} T_{2} T_{1}$ are $c(-1,-1,1)$, $c(1,-1,-1)$ or $c(-1,1,-1)$, respectively; the three other products yield the negatives of these vectors. Hence the six products are translations in $c \Lambda_{(1,1,1)}$.

Now suppose we have an element $R:=T_{j_{1}} T_{j_{2}} \cdots T_{j_{n}}$ of $N$, with $j_{m}=1,2$ or 3 for each $m$. For a non-trivial translation we must have $n \geqslant 3$. If $n \geqslant 3$ and $j_{n-2}, j_{n-1}, j_{n}$ are distinct, then we can reduce $R$ modulo $c \Lambda_{(1,1,1)}$ to a product with $n-3$ terms $T_{j_{m}}$ (by splitting off the product of the last three generators), and then proceed by induction. If $n \geqslant 3$ and $j_{n-2}, j_{n-1}, j_{n}$ are not mutually distinct, then two cases are possible. If $j_{n-1}=j_{n}$, then we directly eliminate the last two generators. If $j_{n-1} \neq j_{n}$, we first insert the trivial element $T_{k} T_{k}$ with $k \neq j_{n-1}, j_{n}$ into the product between $T_{j_{n-2}}$ and $T_{j_{n-1}}$, and 
then split off the product of the last three terms as before. In any case, by induction we can reduce $R$ modulo $c \Lambda_{(1,1,1)}$ to a product with at most two terms, and hence to the empty product if $R$ is a translation.

Now it follows that each translation in $G$ necessarily belongs to $c \Lambda_{(1,1,1)}$.

Note that Lemma 4.2 can be rephrased as

$$
G_{1}(a, b) / c \Lambda_{(1,1,1)} \cong[3,3]^{+} \cong A_{4},
$$

where each term represents the special group. Observe also that the translation subgroup only depends on the single parameter $c=b-a$.

Next we determine the vertex-stars of $P_{1}(a, b)$. The vertex-star at a vertex $x$ of the polyhedron is a (cyclically ordered) set consisting of the vectors $y-x$, where $y$ runs through the (cyclically ordered) vertices of the vertex-figure at $x$. In particular, the vertex-star at $o$ is given by

$$
V_{0}:=\{(a, 0, b),(0, b, a),(b, a, 0)\} .
$$

If $R$ is any element of $G=G_{1}(a, b)$ and again $R^{\prime}$ is its image in the special group $G_{0}$, then the vertex-star at the vertex $o R$ of $P_{1}(a, b)$ is given by

$$
V_{0} R^{\prime}=\left\{(a, 0, b) R^{\prime},(0, b, a) R^{\prime},(b, a, 0) R^{\prime}\right\} .
$$

It is easy to see that the union of all these vertex-stars comprises the set of (generally 12) vectors

$$
V:=\{( \pm a, 0, \pm b),( \pm b, \pm a, 0),(0, \pm b, \pm a)\},
$$

which is the same set as for the polyhedron $P(a, b)$ from which $P_{1}(a, b)$ is derived by $\varphi_{2}$ (see (5.9) of [24] and Fig. 4.1). The points in $V$ are the vertices of a convex 3-polytope, which is combinatorially equivalent to an icosahedron if $a, b \neq 0$ and $a \neq \pm b$, or is a cuboctahedron if $a= \pm b \neq 0$, or an octahedron if $a=0$ or $b=0$.

Now we can find a full set of vertex-stars for $P_{1}(a, b)$. In fact, since $G$ acts transitively on the vertices of $P_{1}(a, b)$, the vertex-stars of $P_{1}(a, b)$ are images of $V_{0}$ under the special

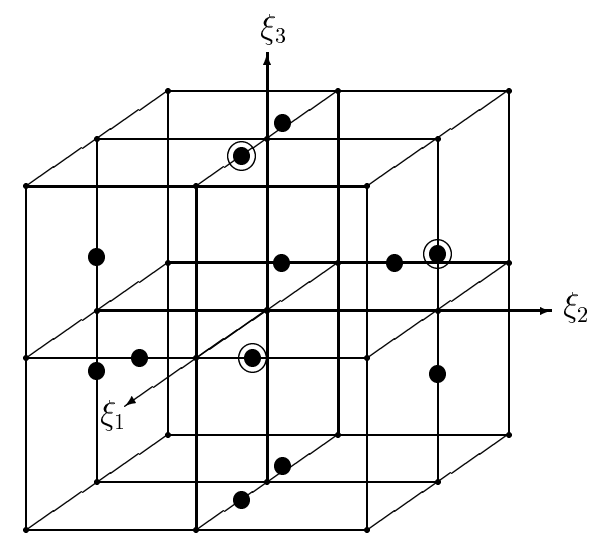

Fig. 4.1. The set $V$ for $a=1$ and $b=3$. The points in the vertex-star $V_{0}$ for $P_{1}(a, b)$ are circled. The cubes are drawn in for reference. 
group $G_{0}\left(\cong A_{4}\right)$, so $G_{0}$ must act transitively on them. However, since $G_{0}$ contains $S_{2}$, the stabilizer of $V_{0}$ must be at least of order 3 , and hence the number of vertex-stars cannot exceed 4 . In fact, there are precisely four vertex-stars (even if $a=0$ or $b=0$ ), namely $V_{0}$ of (4.13) and

$$
\begin{aligned}
& V_{1}:=V_{0} T_{1}^{\prime}=\{(a, 0,-b),(0,-b,-a),(b,-a, 0)\} \\
& V_{2}:=V_{0} T_{2}^{\prime}=\{(-a, 0,-b),(0, b,-a),(-b, a, 0)\} \\
& V_{3}:=V_{0} T_{3}^{\prime}=\{(-a, 0, b),(0,-b, a),(-b,-a, 0)\}
\end{aligned}
$$

They occur as vertex-stars at the vertices $o, o T_{1}=(0, b, a), o T_{2}=(a, 0, b)$ and $o T_{3}=$ $(b, a, 0)$, respectively. Each vector in $V$ lies in exactly one or exactly two vertex-stars according as $a, b \neq 0$, or $a=0$ or $b=0$.

Recall that the edge-module $\Lambda$ of a polyhedron $P$, with $o$ among its vertices, is defined by

$$
\Lambda:=\langle x-y| x, y \text { adjacent vertices of } P\rangle_{\mathbb{Z}}
$$

(see [24]). Then $\Lambda$ is the $\mathbb{Z}$-module generated by the "oriented" edges of $P$; alternatively, $\Lambda$ is the $\mathbb{Z}$-module generated by all the vertex-stars of $P$. If $V(P)$ denotes the vertex-set of $P$, then

$$
V(P) \subset \Lambda
$$

because $P$ is connected. Note that the special group of $G(P)$ leaves $\Lambda$ invariant.

Now let $\Lambda:=\mathbb{Z}[V]$ denote the $\mathbb{Z}$-module spanned by the vectors in $V$. This is the edge-module of $P_{1}(a, b)$ (and $P(a, b)$ ) and has rank at most 12. We proved in Lemma 5.2 of [24] that $\Lambda$ is a (discrete) lattice if and only if $a$ or $b$ is zero or $a$ and $b$ are rational multiples of each other, or, equivalently, if $a, b$ can be scaled to a pair of integers which are relatively prime. On the other hand, if $\Lambda$ is not a lattice, then $\Lambda$ is dense in $\mathbb{E}^{3}$. This follows from general structure results for $\mathbb{Z}$-modules embedded in euclidean spaces (see p. 265 of [27]); bear in mind that $\Lambda$ cannot have a discrete component, because such a component would have to be invariant under the (irreducible) special group $[3,3]^{+}$. For example, if $a=1$ and $b=\tau$, the golden ratio, then $\Lambda$ is the (dense) $\mathbb{Z}$-module spanned by the vertex-set $V$ of a regular icosahedron; similar $\mathbb{Z}$-modules have been studied in the context of icosahedral quasicrystals (see [3]).

We now determine the vertex-set of $P_{1}(a, b)$. Since $P_{1}(a, b)=P(a, b)^{\varphi_{2}}$ and $P_{1}(a, b)$ and $P(a, b)$ share the same base vertex and base edge, the vertices and edges, respectively, of $P_{1}(a, b)$ are among the vertices and edges of $P(a, b)$. However, by (4.8), the vertexstars for $P_{1}(a, b)$ consist only of every other vector in the corresponding vertex-star for $P(a, b)$. This indicates that the vertex-set and edge-set of $P_{1}(a, b)$ are generally only "thin" subsets of those of $P(a, b)$. In fact, we have

Lemma 4.3. The vertex-set of $P_{1}(a, b)$ is given by

$$
V\left(P_{1}(a, b)\right)=\{(0,0,0),(a, 0, b),(0, b, a),(b, a, 0)\}+c \Lambda_{(1,1,1)},
$$

with $c:=b-a$, where the four cosets of $c \Lambda_{(1,1,1)}$ occurring are distinct. In particular, the vertex-set is discrete (it is finite when $b=a$ ). 
Proof. Set $T_{0}:=I$. It follows from (4.12) and the proof of Lemma 4.2 that the 12 elements

$$
S_{2}^{j} T_{k} \quad(j=0,1,2 ; k=0,1,2,3)
$$

form a system of representatives of $G$ modulo $c \Lambda_{(1,1,1)}$. Hence the vertex-set is the union of the 12 cosets $o S_{2}^{j} T_{k}+c \Lambda_{(1,1,1)}$. However, since $S_{2}$ fixes the base vertex $o$, there are only four cosets, namely $o T_{k}+c \Lambda_{(1,1,1)}$ with $k=0,1,2,3$. Thus the vertex-set consists of the cosets of $o$ and its adjacent vertices. Moreover, these four cosets are distinct. In fact, if $(a, 0, b) \equiv(0,0,0)$ (say) modulo $c \Lambda_{(1,1,1)}$, then $(a / c, 0, b / c)=(a / c, 0,1+a / c) \in$ $\Lambda_{(1,1,1)}$, so $a / c$ and $1+a / c$ must both be even integers; however, this is impossible. This proves the lemma.

Note that the vertex-set of $P_{1}(a, b)$ is discrete, irrespective of the values of $a$ and $b$. By contrast, if $a$ and $b$ are non-zero and are not rational multiples of each other, then the vertex-set of $P(a, b)$ is dense in $\mathbb{E}^{3}$; in fact, since $2 \Lambda$ is a subgroup of the translation group of $P(a, b)$, the vertex-set must contain the points in $2 \Lambda$ (see (5.13) of [24]).

Lemma 4.3 also enables us to find the vertex-star at a given vertex $x$ of $P_{1}(a, b)$. In fact, since $c \Lambda_{(1,1,1)}$ is the translation subgroup, the vertex-star at $x$ is the same as the vertex-star at the representative vertex of $x+c \Lambda_{(1,1,1)}$ listed in the lemma. In other words, first determine which vertex among $(0,0,0),(a, 0, b),(0, b, a),(b, a, 0)$ is equivalent to $x$ modulo $c \Lambda_{(1,1,1)}$, and then take its vertex-star, $V_{j}$ (say). The vertices of $P_{1}(a, b)$ adjacent to $x$ are then the points in $x+V_{j}$. Recall that the vertex-stars at $(0,0,0),(a, 0, b)$, $(0, b, a)$ or $(b, a, 0)$ are given by $V_{0}, V_{2}, V_{1}$ or $V_{3}$, respectively, so we have $j=0,2,1$ or 3 .

Lemma 4.4. $\quad P_{1}(a, b)$ is a geometric polyhedron.

Proof. We have altogether four translation classes of vertices and four vertex-stars, and the vertex-stars at vertices of distinct translation classes are also distinct. Hence each translation class of vertices is characterized by its unique vertex-star. It follows that $P_{1}(a, b)$ cannot have multiple vertices; in other words, $P_{1}(a, b)$ must be a faithful realization of the underlying abstract polyhedron. We can also see this more directly as follows. Let $x$ be a vertex of $P_{1}(a, b)$ with vertex-star $V_{j}$, and let $y$ be a vertex adjacent to $x$ such that $y-x \in V_{j}$. We need to show that $x-y$ belongs to the vertex-star $V_{k}$ (say) at $y$, so that $x \in y+V_{k}$. Since we can reduce $x$ modulo $c \Lambda_{(1,1,1)}$, we may assume that $x$ is among $(0,0,0),(a, 0, b),(0, b, a)$ or $(b, a, 0)$. However, now it is straightforward to verify that the corresponding adjacent vertices and their vertex-stars have the desired property.

Notice that our methods also provide an alternative description of the edge-graph of $P_{1}(a, b)$ which does not appeal to the group. In fact, take the set of points described in Lemma 4.3 as the vertex-set of the graph and place, at each vertex $x$, the corresponding vertex-star from the list found by reducing $x$ modulo $c \Lambda_{(1,1,1)}$. Then the resulting graph coincides with the edge-graph of $P_{1}(a, b)$.

The vertex-figures of $P_{1}(a, b)$ are triangles. However, the vertex-stars of $P_{1}(a, b)$ are generally not planar. In fact, we have the following: 
Lemma 4.5. The vertex-stars of $P_{1}(a, b)$ are planar if and only if $b=-a$.

Proof. The special group $G_{0}$ acts transitively on the vertex-stars. The three vectors in $V_{0}$ have determinant $\pm\left(a^{3}+b^{3}\right)$, so they lie in a plane if and only if $b=-a$.

Next we determine which polyhedra are regular.

Lemma 4.6. The polyhedron $P_{1}(a, b)$ is geometrically chiral if $b \neq \pm a$, or geometrically regular if $b= \pm a$. In particular, $P_{1}(a,-a)$ is similar to $P_{1}(1,-1)=\{\infty, 3\}^{(a)}$, and $P_{1}(a, a)$ is similar to $P_{1}(1,1)=\{3,3\}$.

Proof. The case $b= \pm a$ was already settled (see (4.9) and (4.10)), so we may assume that $b \neq \pm a$. Suppose $P_{1}(a, b)$ is regular. Then $P_{1}(a, b)$ must have an involutory symmetry $R\left(=R_{2}\right)$ which fixes $o$ and $v$ and interchanges the neighbors $(0, b, a)$ and $(b, a, 0)$ of $o$ (see also Lemma 2.1). Since $V_{0}$ is not planar, $R$ must necessarily be the (linear) reflection in the plane through $o, v$ with normal vector

$$
n:=(0, b, a)-(b, a, 0)=(-b, b-a, a) .
$$

Moreover, since $R^{-1} T R=T$, the rotation axis of $T$ must be invariant under $R$. However, the direction vector $e_{2}=(0,1,0)$ of this axis is not a scalar multiple of $n$, so $e_{2}$ must lie in the mirror of $R$. Hence $b-a=n \cdot e_{2}=0$ (where denotes the scalar product), which contradicts our assumption on $a, b$. (Recall that $R_{2}$ is a half-turn for $\{\infty, 3\}^{(a)}$, so a contradiction can only concern the case $b=a$.) Now the lemma follows.

The two enantiomorphic forms of a chiral polyhedron are represented by different pairs of generators of its group. If $S_{1}(a, b), S_{2}$ is the pair associated with the base flag $\Phi(a, b):=\left\{F_{0}(a, b), F_{1}(a, b), F_{2}(a, b)\right\}$ of $P_{1}(a, b)$, then $S_{1}(a, b) S_{2}^{2}, S_{2}^{-1}$ is the pair associated with the adjacent flag $\Phi^{2}(a, b):=\left\{F_{0}(a, b), F_{1}(a, b), F_{2}^{\prime}(a, b)\right\}$ of $P_{1}(a, b)$. Since the product of each pair is $T(a, b)$, we can also represent the two forms by the pairs of generators $T(a, b), S_{2}$ and $T(a, b), S_{2}^{-1}$, respectively. When Wythoff's construction is applied to $G_{1}(a, b)$ with the new pair of generators $T(a, b), S_{2}^{-1}$ and with the same initial vertex $o$, which is also fixed by $S_{2}^{-1}$, then we again obtain the same polyhedron $P_{1}(a, b)$, but now with a new base flag adjacent to the original base flag. We can see this as follows.

First we claim that

$$
P_{1}(b, a)=P_{1}(a, b) R,
$$

where $R$ is the reflection (in the plane $\xi_{1}=\xi_{3}$ ) given by

$$
R:\left(\xi_{1}, \xi_{2}, \xi_{3}\right) \mapsto\left(\xi_{3}, \xi_{2}, \xi_{1}\right) .
$$

In fact, conjugation by $R$ transforms the generators $T(a, b), S_{2}$ for $G_{1}(a, b)$ to the generators $T(b, a), S_{2}^{-1}$ for $G_{1}(b, a)$, so in particular $G_{1}(b, a)=R^{-1} G_{1}(a, b) R$. Then

$$
V\left(P_{1}(b, a)\right)=o G_{1}(b, a)=o R^{-1} G_{1}(a, b) R=o G_{1}(a, b) R=V\left(P_{1}(a, b)\right) R,
$$


so $R$ maps the vertex-sets of the polyhedra onto each other. Similarly, since

$$
F_{1}(b, a) G_{1}(b, a)=F_{1}(b, a) R^{-1} G_{1}(a, b) R=\left(F_{1}(a, b) G_{1}(a, b)\right) R,
$$

the same holds for the edge-sets. Finally, $R$ takes $F_{2}(b, a)$ to the face $F_{2}(a, b)^{\prime}$ (= $\left.F_{2}(a, b) T(a, b)\right)$ of $\Phi^{2}(a, b)$ (see (4.4)), so the corresponding statement for face-sets follows from

$$
F_{2}(b, a) G_{1}(b, a)=F_{2}(b, a) R^{-1} G_{1}(a, b) R=\left(F_{2}(a, b)^{\prime} G_{1}(a, b)\right) R .
$$

This establishes (4.17). Moreover, notice that

$$
\Phi(b, a) R=\Phi^{2}(a, b), \quad \Phi^{2}(b, a) R=\Phi(a, b) .
$$

Now we can complete our argument about enantiomorphism. In fact, bearing in mind how conjugation by $R$ affects the generators we observe that the polyhedron $P$ (say) obtained by Wythoff's construction from $G_{1}(a, b)$ with generators $T(a, b), S_{2}^{-1}$, is mapped by $R$ to the polyhedron obtained from $G_{1}(b, a)$ with generators $T(b, a), S_{2}$. (Note that we interchanged $a$ and $b$.) However, the latter is just $P_{1}(b, a)$, so by (4.17) its preimage under $R$ is $P_{1}(a, b)$ itself. Hence, $P=P_{1}(a, b)$.

These considerations also justify our initial hypothesis that it suffices to concentrate on only one orientation for the generator $S_{2}$. The opposite orientation (given by $S_{2}^{-1}$ ) then is implied by enantiomorphism.

Alternatively we could have appealed to similar such results for the polyhedra $P(a, b)$, by observing that conjugation by $R$ commutes with the facetting operation $\varphi_{2}$ (see $(5.18)$ of [24]). In particular,

$$
(P(a, b) R)^{\varphi_{2}}=\left(P(a, b)^{\varphi_{2}}\right) R .
$$

We now discuss the question when two polyhedra $P_{1}(a, b)$ and $P_{1}(c, d)$ with parameter sets $a, b$ and $c, d$, respectively, are affine images of each other. The result is consistent with Lemma 5.15 of [24], which describes the answer for the polyhedra $P(a, b)$ and $P(c, d)$.

Lemma 4.7. Let $a, b, c, d$ be real numbers, and let $(a, b) \neq(0,0) \neq(c, d)$. Then the polyhedra $P_{1}(a, b)$ and $P_{1}(c, d)$ are affinely equivalent if and only if $(c, d)=s(a, b)$ or $(c, d)=t(b, a)$ for some non-zero scalars $s$ or $t$. Moreover, $P_{1}(a, b)$ and $P_{1}(c, d)$ are congruent if and only if $(c, d)= \pm(a, b)$ or $(c, d)= \pm(b, a)$.

Proof. We already know that the two polyhedra are affinely equivalent if $(c, d)=$ $s(a, b)$ or $t(b, a)$ (see (4.5) and (4.17)).

Suppose $S$ is an affine transformation of $\mathbb{E}^{3}$ such that $P_{1}(c, d)=P_{1}(a, b) S$. Since $G_{1}(c, d)$ acts transitively on the vertices, and the stabilizer of the vertex $o$ acts transitively on the vertices adjacent of $o$, we can assume that $o S=o$ (so $S$ is linear) and $(a, 0, b) S=$ $(c, 0, d)$. First observe that $S$ and $S^{-1}$ preserve planarity of vertex-stars, so certainly $b=-a$ if and only if $d=-c$; in this case the assertion is obvious. Let $b \neq-a$, so now the vertex-stars are not planar. The affine transformation $S^{-1} T(a, b) S$ interchanges the vertices in the base edge of $P_{1}(c, d)$, as well as the two faces meeting at this base edge. 


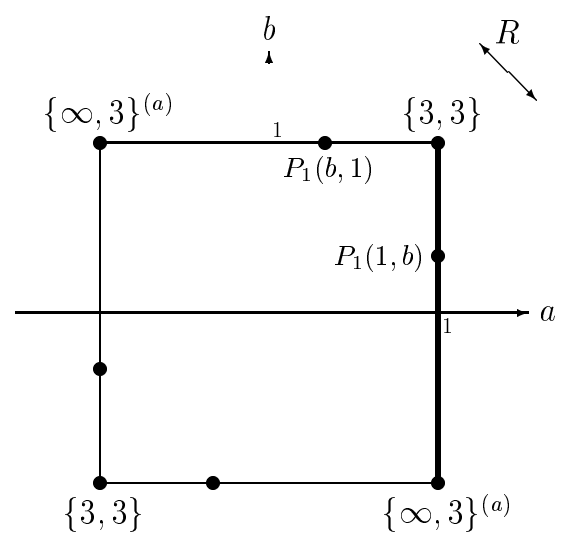

Fig. 4.2. The affine classes of polyhedra $P_{1}(a, b)$.

Since this is also true for the symmetry $T(c, d)$ of $P_{1}(c, d)$, we must have $S^{-1} T(a, b) S=$ $T(c, d)$; recall that an affine transformation is uniquely determined by its effect on four independent points. Similarly, $S^{-1} S_{2} S$ cyclically permutes the vertices in the vertexfigure of $P_{1}(c, d)$ at $o$, so we must also have $S^{-1} S_{2} S=S_{2}$ or $S_{2}^{-1}$. These properties for the generators translate into conditions for the matrix entries of $S$ and show that, up to similarity, there are only the following two possibilities. If $S^{-1} S_{2} S=S_{2}$, then $S=s I$ for some scalar $s$, and hence $(c, d)=s(a, b)$. If $S^{-1} S_{2} S=S_{2}^{-1}$, then $S=t R$ with $R$ as in (4.18), for some scalar $t$; hence $(c, d)=t(b, a)$. Moreover, if $S$ is an isometry, then necessarily $s= \pm 1$ or $t= \pm 1$.

Figure 4.2 illustrates the situation described in Lemma 4.7. Each affine equivalence class of polyhedra is represented by a polyhedron $P_{1}(1, b)$ corresponding to the point $(1, b)$ on the right side of the square. The equivalence classes of the regular polyhedra $\{3,3\}$ and $\{\infty, 3\}^{(a)}$ then occur at the upper right corner and lower right corner, respectively. The other three sides of the square give alternative representations of the equivalence classes, as indicated by the four symmetrically related nodes.

The group $G_{1}(a, b)$ associated with $P_{1}(a, b)$ is generated by rotations and thus contains only direct isometries. This implies that the faces of $P_{1}(a, b)$ consist of either all right-hand helices or all left-hand helices. Note that the faces of $P_{1}(a, b)$ are right-hand helices if and only if the faces of $P_{1}(b, a)$ are left-hand helices; in fact, the plane reflection $R$ of (4.18) maps $P_{1}(a, b)$ to $P_{1}(b, a)$, and vice versa. When $b=-a$, these polyhedra are regular and congruent, but they do not coincide (so $R$ is not a symmetry); in fact, every symmetry of $\{\infty, 3\}^{(a)}$ is a direct isometry. On the other hand, if $b=a$, then $R$ is indeed a symmetry.

We conclude this section with a discussion of the translation classes of faces of $P_{1}(a, b)$, by which we mean the transitivity classes of faces under the translation subgroup $c \Lambda_{(1,1,1)}$ of $G=G_{1}(a, b)$, where again $c:=b-a$. When $P_{1}(a, b)$ is regular, no translational symmetries in addition to those in $G_{1}(a, b)$ occur, so the translation classes with respect to the full symmetry group of $P_{1}(a, b)$ are the same. 


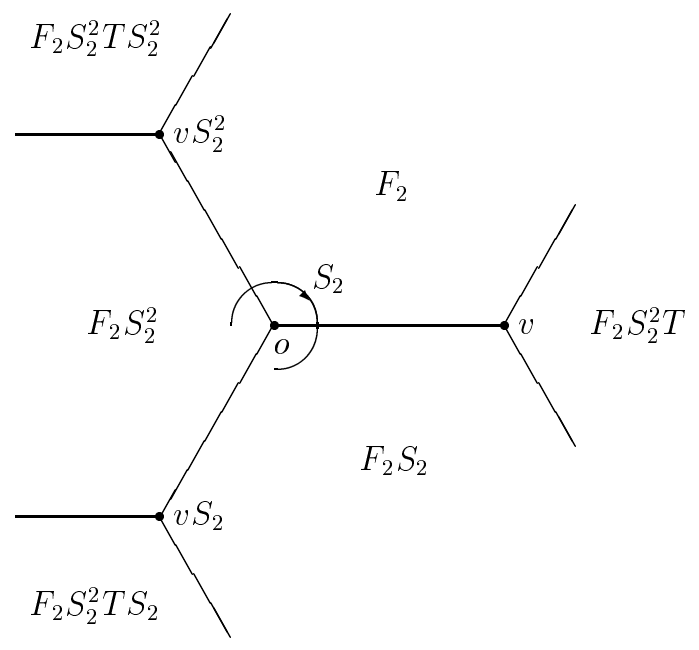

Fig. 4.3. The faces near $o$.

Lemma 4.8. $\quad P_{1}(a, b)$ has precisely four translation classes of faces. They are represented by the four faces $F_{2}, F_{2} S_{2}, F_{2} S_{2}^{2}$ and $F_{2} S_{2}^{2} T$, where $F_{2}$ is the base face given by (4.4).

Proof. By Lemma 4.3 each vertex of $P_{1}(a, b)$ is equivalent to $o, v, v S_{2}$ or $v S_{2}^{2}$ modulo $c \Lambda_{(1,1,1)}$. Hence, modulo $c \Lambda_{(1,1,1)}$, every face of $P_{1}(a, b)$ is equivalent to a face containing one of these vertices. There are six such faces, including the three which contain $o$, namely $F_{2}, F_{2} S_{2}\left(=F_{2}^{\prime}\right)$ and $F_{2} S_{2}^{2}$. The other three faces, $F_{2} S_{2}^{2} T, F_{2} S_{2}^{2} T S_{2}$ and $F_{2} S_{2}^{2} T S_{2}^{2}$, contain the vertices $v, v S_{2}$ and $v S_{2}^{2}$, respectively. The combinatorial picture is illustrated in Fig. 4.3. In the first set of three, the helical faces have an axis with direction vector $(1,-1,-1),(-1,-1,1)$ or $(-1,1,-1)$, respectively. However, in the second set, the faces are all equivalent modulo $c \Lambda_{(1,1,1)}$ and have an axis with direction vector $(1,1,1)$. In particular,

$$
F_{2} S_{2}^{2} T S_{2}=F_{2} S_{2}^{2} T+(c, c,-c), \quad F_{2} S_{2}^{2} T S_{2}^{2}=F_{2} S_{2}^{2} T+(2 c, 0,0) .
$$

This concludes the proof.

\section{Type $\{\infty, 3\}$, with Helical Faces over Squares}

In this section we describe another family of chiral apeirohedra of type $\{\infty, 3\}$, now with helical faces over squares. They occur when $G_{0}=[3,4]^{+}$in Lemma 3.6. As in the previous section, each polyhedron is obtained from an irreducible group $G$ by Wythoff's construction with initial vertex $F_{0}:=o$.

For a polyhedron $P$ of type $\{\infty, 3\}$ with helical faces over squares, we must begin with a group $G=\left\langle S_{1}, S_{2}\right\rangle$ with special group $G_{0}=[3,4]^{+}$, where $S_{1}^{\prime}, S_{2}$ are standard generators of $G_{0}$ considered as the group of the cube $\{4,3\}$, so that $S_{1}^{\prime}$ and $S_{2}$ have 
periods 4 and 3, respectively, and their product has period 2. (Recall that $R^{\prime}$ denotes the image in $G_{0}$ of an element $R$ of $G$.) As already mentioned in the proof of Lemma 3.3, once $S_{2}$ has been selected, there are three equivalent choices for $S_{1}^{\prime}$ such that $S_{1}^{\prime} S_{2}$ has period 2; if $S_{1}^{\prime}$ is one of them, then the other two are $S_{2}^{-1} S_{1}^{\prime} S_{2}$ and $S_{2}^{-2} S_{1}^{\prime} S_{2}^{2}$. Moreover, substituting $S_{2}$ by its inverse would lead to (the enantiomorphic image of) the same underlying polyhedron, so we need not discuss this case separately.

As in the previous section, we may confine ourselves to some very specific choices for the generators. Thus, with

$$
\begin{aligned}
& S_{1}^{\prime}: x \mapsto\left(-\xi_{3}, \xi_{2}, \xi_{1}\right), \\
& S_{2}: x \mapsto\left(\xi_{2}, \xi_{3}, \xi_{1}\right),
\end{aligned}
$$

we observe that $T^{\prime}=S_{1}^{\prime} S_{2}$ is the half-turn about the line $\xi_{2}=\xi_{1}$ in the $\xi_{1} \xi_{2}$-plane. Then $T=S_{1} S_{2}$ is a half-turn about a (parallel) line perpendicular to the plane $\xi_{2}=-\xi_{1}$, and since $T$ must interchange the two vertices in the base edge $F_{1}$, this base edge must lie in this plane and the rotation axis of $T$ must pass through its midpoint. In particular, $v:=o T=(c,-c, d)$ for some real parameters $c$ and $d$, not both zero. The resulting group $G=G_{2}(c, d)$ is generated by

$$
\begin{aligned}
S_{2}: \quad x & \mapsto\left(\xi_{2}, \xi_{3}, \xi_{1}\right), \\
T: \quad x & \mapsto\left(\xi_{2}, \xi_{1},-\xi_{3}\right)+(c,-c, d) .
\end{aligned}
$$

For $S_{1}=T S_{2}^{-1}$ we have

$$
\begin{aligned}
& S_{1}: \quad x \quad \mapsto \quad\left(-\xi_{3}, \xi_{2}, \xi_{1}\right)+(d, c,-c), \\
& S_{1}^{4}: \quad x \mapsto\left(\xi_{1}, \xi_{2}, \xi_{3}\right)+4 c(0,1,0) .
\end{aligned}
$$

The latter is the translation by $4 c(0,1,0)$, and is trivial if $c=0$. The special case $c=0$ yields the finite group

$$
G_{2}(0, d) \cong[3,4]^{+}
$$

(with fixed point $\frac{1}{2}(d, d, d)$ ); the corresponding polyhedron is a cube.

The polyhedron $P=P_{2}(c, d)$ is obtained from $G=G_{2}(c, d)$ by Wythoff's construction with base vertex $F_{0}=o$. Its base edge $F_{1}$ has vertices $o$ and $v=(c,-c, d)$; its base face is given by

$$
F_{2}=o\left\langle S_{1}\right\rangle=\{(c,-c, d),(0,0,0),(d, c,-c),(c+d, 2 c,-c+d)\}+\mathbb{Z} \cdot 4 c(0,1,0)
$$

and consists of a helix over a square. (The notation for helical faces is as before, but now there are four consecutive vertices that are translated.) As usual, the remaining vertices, edges and faces of $P$ are the images of $F_{0}, F_{1}$ and $F_{2}$ under $G$. The vertices adjacent to $o$ are

$$
v=(c,-c, d), \quad v S_{2}=(-c, d, c), \quad v S_{2}^{2}=(d, c,-c) .
$$

When the polyhedra are only considered up to similarity, the two parameters $c, d$ reduce to a single parameter. In fact, (4.5) carries over and shows that $P_{2}(c, d)$ is similar to $P_{2}(1, d / c)$ or $P_{2}(0,1)$ according as $c \neq 0$ or $c=0$. 
As in the previous section, the facetting operation $\varphi_{2}$ establishes a link to polyhedra with finite faces (equivalently, we can employ the operation $\kappa$ discussed in Section 8). Now the polyhedra $P_{2}(c, d)$ are related to the chiral (or regular) polyhedra $Q(c, d)$ of type $\{4,6\}$ described in Section 6 of [24]; the latter generally have skew square faces and skew hexagonal vertex-figures. Recall that the generators $\widehat{S}_{1}, \widehat{S}_{2}$ (say) and corresponding element $\widehat{T}:=\widehat{S}_{1} \widehat{S}_{2}$ of the symmetry group $H(c, d)$ of $Q(c, d)$ are given by

$$
\begin{aligned}
& \widehat{S}_{1}: x \mapsto\left(-\xi_{1}, \xi_{3},-\xi_{2}\right)+(c,-d,-c), \\
& \widehat{S}_{2}: \quad x \mapsto-\left(\xi_{3}, \xi_{1}, \xi_{2}\right), \\
& \widehat{T}: \quad x \mapsto\left(\xi_{2}, \xi_{1},-\xi_{3}\right)+(c,-c, d) .
\end{aligned}
$$

Now we have

Lemma 5.1. $\quad P_{2}(c, d)=Q(c, d)^{\varphi_{2}}$, for all real parameters $c$ and $d$.

Proof. In terms of the generators $\widehat{S}_{2}, \widehat{T}$ of $H(c, d)$, the operation $\varphi_{2}$ takes the same form as in (4.8). Now $P_{2}(c, d)=Q(c, d)^{\varphi_{2}}$ and $G_{2}(c, d)$ is a subgroup of $H(c, d)$.

This relationship holds again without any restriction on the parameters, so in particular we may (and often do) have the situation that $P_{2}(c, d)$ is discrete but $Q(c, d)$ is not. Moreover, the lemma implies that the faces of $P_{2}(c, d)$ are among the holes of $Q(c, d)$, which now are helices over squares, except when $c=0$ (then they are squares).

The facetting operation $\varphi_{2}$ preserves regularity, so again there are two special cases. From $Q(1,0)=\{4,6\}_{6}$ we obtain the regular polyhedron

$$
P_{2}(1,0)=\{\infty, 3\}^{(b)}
$$

(notation as on p. 224 of [20]); all other polyhedra $P_{2}(c, 0)$ are similar to $P_{2}(1,0)$. Similarly, the case $c=0$ reduces to $(c, d)=(0,1)$ and again yields regular polyhedra. Now $Q(0,1)=\{4,6 \mid 4\}$ and $\{4,6 \mid 4\}^{\varphi_{2}}=\{4,3\}$ (see Theorem 6.12 of [24] and p. 224 of [20]), so in particular

$$
P_{2}(0,1)=\{4,3\}
$$

Next we determine the translation subgroup $T(G)$ of $G=G_{2}(c, d)$. Since $S_{1}^{4}$ is the translation by $4 c(0,1,0)$, and its conjugates under the special group $G_{0}\left(\cong[3,4]^{+}\right)$ consist of the vectors $\pm 4 c(1,0,0), \pm 4 c(0,1,0)$ and $\pm 4 c(0,0,1)$, we immediately see that

$$
4 c \mathbb{Z}^{3}=4 c \Lambda_{(1,0,0)} \leqslant T(G) .
$$

In fact, we have

Lemma 5.2. The translation subgroup of $G_{2}(c, d)$ is given by $T\left(G_{2}(c, d)\right)=4 c \mathbb{Z}^{3}$.

Proof. The proof is more involved than the proof of Lemma 4.2 and is interesting in its own right. As before, since $G=\left\langle S_{2}, T\right\rangle$, we have $G=N \cdot\left\langle S_{2}\right\rangle$ (as a product of subgroups), where $N:=\left\langle T_{1}, T_{2}, T_{3}\right\rangle$ with $T_{2}:=T, T_{1}:=S_{2}^{-1} T S_{2}$ and $T_{3}:=S_{2}^{-2} T S_{2}^{2}$. 
Now $S_{2}$ is the square of the generator $\widehat{S}_{2}$ for the group $H(c, d)$ of $Q(c, d)$ (see (4.8) and (5.5)), so $T_{1}, T_{2}, T_{3}$ are just the half-turns occurring in Section 6 of [24] with the same notation, namely

$$
\begin{aligned}
& T_{1}: \quad x \mapsto\left(\xi_{3},-\xi_{2}, \xi_{1}\right)+(-c, d, c), \\
& T_{2}: \quad x \mapsto\left(\xi_{2}, \xi_{1},-\xi_{3}\right)+(c,-c, d), \\
& T_{3}: \quad x \mapsto\left(-\xi_{1}, \xi_{3}, \xi_{2}\right)+(d, c,-c) .
\end{aligned}
$$

It is straightforward to check that $\left(T_{i} T_{j}\right)^{3}$ and $\left(T_{i} T_{j} T_{k}\right)^{4}$, but not $T_{i} T_{j}$ or $\left(T_{i} T_{j} T_{k}\right)^{2}$, are translations in $4 c \mathbb{Z}^{3}$, for all mutually distinct $i, j, k$.

We now consider the six elements $T_{i} T_{j} T_{k} T_{j}$ of $N$ with $i, j, k$ distinct. They are

$$
\begin{array}{llll}
U_{1}:=T_{1} T_{2} T_{3} T_{2}, & U_{2}:=T_{2} T_{1} T_{3} T_{1}, & U_{3}:=T_{3} T_{1} T_{2} T_{1}, \\
\widehat{U}_{1}:=T_{1} T_{3} T_{2} T_{3}, & \widehat{U}_{2}:=T_{2} T_{3} T_{1} T_{3}, & \widehat{U}_{3}:=T_{3} T_{2} T_{1} T_{2} .
\end{array}
$$

In particular, we have

$$
\begin{aligned}
& U_{1}: \quad x \quad \mapsto \quad\left(-\xi_{1}, \xi_{2},-\xi_{3}\right)+(c+d,-2 c, 3 c+d), \\
& U_{2}: \quad x \mapsto\left(-\xi_{1},-\xi_{2}, \xi_{3}\right)+(-c+d,-3 c+d, 2 c), \\
& U_{3}: \quad x \mapsto\left(\xi_{1},-\xi_{2},-\xi_{3}\right)+(-2 c, 3 c+d, c+d) .
\end{aligned}
$$

Then $U_{i}^{2}, \widehat{U}_{i}^{2}$ and $U_{i} \widehat{U}_{i}$ are translations in $4 c \mathbb{Z}^{3}$, for each $i$. Now define the subgroups

$$
K:=\left\langle U_{1}, U_{2}, U_{3}\right\rangle, \quad K^{2}:=\left\langle U_{1}^{2}, U_{2}^{2}, U_{3}^{2}\right\rangle
$$

of $N$. Then it is easy to see that $4 c \mathbb{Z}^{3}=K^{2} \leqslant K$, and hence also $\widehat{U}_{1}, \widehat{U}_{2}, \widehat{U}_{3} \in K$. In particular, modulo $K^{2}$, each element $U_{i}$ has period 2 and coincides with $\widehat{U}_{i}$. Moreover, each product $U_{i} U_{j} U_{k}$, with $i, j, k$ distinct, is a translation in $K^{2}$.

Next observe that $S_{2} \in N$, so in particular $N=G$. In fact, $S_{1} T_{1} U_{3}$ is a translation in $K^{2}$, so

$$
S_{1} \in K^{2} U_{3} T_{1} \subseteq K T_{1} \subseteq N .
$$

Hence also $S_{2}=S_{1}^{-1} T_{2} \in N$.

Now, modulo $K$, each element of $G$ can be represented by an element (a right coset representative) which is a product of at most three generators $T_{i}$. In fact, if $\varphi=T_{i_{1}} T_{i_{2}} \cdots T_{i_{n}} \in G$, then we can split off elements of the form $T_{i} T_{j} T_{k} T_{j}$ and proceed inductively; for example, if $i_{1}, i_{2}, i_{3}$ are distinct, then insert the trivial product $T_{i_{2}} T_{i_{2}}$ between the third and fourth term of $\varphi$, split off $T_{i_{1}} T_{i_{2}} T_{i_{3}} T_{i_{2}}$, and proceed with $T_{i_{2}} T_{i_{4}} \cdots T_{i_{n}}$.

Moreover, modulo $K^{2}$, each element of $K$ can be represented by $I, U_{1}, U_{2}$ or $U_{3}$ (as right coset representatives), so in particular the subgroup $K^{2}$ has index 4 in $K$. In fact, suppose $\psi=U_{i_{1}} U_{i_{2}} \cdots U_{i_{m}} \in K$; modulo $K^{2}$, each generator has period 2, so we need not consider any product involving their inverses. In this case we can split off elements of the form $U_{i} U_{j} U_{k}$ and again proceed inductively; for example, if $i_{1}, i_{2}$ are distinct and $j \neq i_{1}, i_{2}$, then insert the (trivial, modulo $K^{2}$ ) product $U_{j} U_{j}$ between the second and third term of $\psi$, split off $U_{i_{1}} U_{i_{2}} U_{j}$, and proceed with $U_{j} U_{i_{3}} \cdots U_{i_{m}}$. This, then, reduces $\psi$ modulo $K^{2}$ to an element which is a product of at most two generators $U_{i}$. If in fact this product involves two generators and is given by $U_{i_{1}} U_{i_{2}}$ (say), then we can further reduce it to $U_{j}$ with $j \neq i_{1}, i_{2}$, by multiplying by $U_{j} U_{j}$ on the right and again splitting off $U_{i_{1}} U_{i_{2}} U_{j}$. 
Now it follows that each translation in $K$ already lies in $K^{2}$; that is, $T(G) \cap K=K^{2}$. In fact, none of the elements $U_{1}, U_{2}, U_{3}$ is a translation, so any translation in $K$ must be equivalent to $I$ modulo $K^{2}$. Note that this also shows that $K^{2}$ is normal in $K$. In particular, $K / K^{2} \cong C_{2} \times C_{2}$, with $C_{2} \times C_{2}$ generated by any two of the generators $U_{i}$.

We now complete the proof by considering images of elements in the special group $G_{0}$. First observe that, since this is true for the generators, each element of $K$, considered in the special group, takes the form $x \mapsto\left( \pm \xi_{1}, \pm \xi_{2}, \pm \xi_{3}\right)$, with exactly two minus signs or no minus sign (for the trivial element); in particular, no permutation of coordinates occurs. On the other hand, modulo $K$, each element of $G$ can be represented by an element which is a product of at most three generators $T_{i}$, and it is not hard to see that in fact the six elements

$$
I, T_{1}, T_{2}, T_{3}, T_{1} T_{2}, T_{2} T_{1}
$$

suffice and give a complete system of right coset representatives of $G$ modulo $K$. However, the elements in each coset of $K$ distinct from $K$ all involve a (in fact, the same) non-trivial permutation of the coordinates, because this is true for the corresponding representatives. Hence no such coset can contain a translation, because any translation is trivial in the special group. Now it follows that $T(G)=4 c \mathbb{Z}^{3}$.

An alternative system of representatives of $G$ modulo $K$ consists of the six elements

$$
I, T_{1}, S_{2}, T_{1} S_{2}, S_{2}^{2}, T_{1} S_{2}^{2},
$$

which turns out to be more useful for the vertex-set computation. From this we also obtain a system of right coset representatives for $G$ modulo $4 c \mathbb{Z}^{3}$, by multiplying each of these elements on the left by $I, U_{1}, U_{2}$ or $U_{3}$.

The translation subgroup depends only on a single parameter, $c$. Now Lemma 4.2 can be rephrased as

$$
G_{2}(c, d) / 4 c \mathbb{Z}^{3} \cong[3,4]^{+} \cong S_{4},
$$

with each term representing the special group.

We now compute the vertex-set of $P_{2}(c, d)$. Again the vertex-set is discrete, irrespective of the values of the parameters $c$ and $d$.

Lemma 5.3. The vertex-set of $P_{2}(c, d)$ is given by $V\left(P_{2}(c, d)\right)=Z+4 c \mathbb{Z}^{3}$, where $Z$ consists of the points

$$
\left\{\begin{array}{l}
(0,0,0) \\
(c+d, 2 c,-c+d), \quad \text { and its cyclic shifts; } \\
(2 c+d, 2 c+d, 2 c+d) \\
(c,-c, d), \quad \text { and its cyclic shifts. }
\end{array}\right.
$$

Proof. Let $U_{1}, U_{2}, U_{3}$ and $K$ be as in the proof of the previous lemma (see (5.10) and (5.11)). First observe that $o K$, the orbit of the base vertex $o$ under $K$, is the union of the translation classes of the four vertices $o, o U_{1}, o U_{2}, o U_{3}$ modulo $4 c \mathbb{Z}^{3}$, or, equivalently, of the four vertices

$$
(0,0,0), \quad(c+d, 2 c,-c+d), \quad(-c+d, c+d, 2 c), \quad(2 c,-c+d, c+d),
$$


respectively. Then $o K T_{1}$, the image of $o K$ under $T_{1}$, is the union of the translation classes of the four vertices

$$
(-c, d, c), \quad(2 c+d, 2 c+d, 2 c+d), \quad(c,-c, d), \quad(d, c,-c) .
$$

These two sets of four vertices are invariant under $S_{2}$, so their union is also invariant under $S_{2}$. It follows that no additional vertices arise from the images of $o K$ under the elements $S_{2}, T_{1} S_{2}, S_{2}^{2}$ or $T_{1} S_{2}^{2}$, that is, from the remaining cosets $K S_{2}, K T_{1} S_{2}, K S_{2}^{2}$ and $K T_{1} S_{2}^{2}$ of $K$ (see (5.12)). This proves the lemma.

Observe that, when $c=0$, the polyhedron is the cube $P_{2}(0, d)$ with vertex-set $Z$.

For certain parameter values the eight translation classes of vertices may not all be distinct. However, bearing in mind that the set of eight translation classes is invariant under $S_{2}$, inspection shows that two classes can only coincide if $d=k c$ with $k \in \mathbb{Z}$ and $k \equiv 2(\bmod 4)$. This occurs precisely when $(2 c+d, 2 c+d, 2 c+d)$ is equivalent to $(0,0,0)$ modulo $4 c \mathbb{Z}^{3}$, and then $(c,-c, d)$ and $(-c+d, c+d, 2 c)$, as well as their cyclic shifts, are also equivalent modulo $4 c \mathbb{Z}^{3}$. However, no further collapses occur, because $(c,-c, d)$ and its cyclic shifts are not equivalent to $(0,0,0)$ modulo $4 c \mathbb{Z}^{3}$ for any parameter values. This proves

Lemma 5.4. $\quad P_{2}(c, d)$ has eight translation classes of vertices modulo $4 c \mathbb{Z}^{3}$, except when $d=k c$ and $k$ is an integer with $k \equiv 2(\bmod 4)$; they are represented by the vertices listed in Lemma 5.3. If $d=k c$ and $k$ is an integer with $k \equiv 2(\bmod 4)$, then $P_{2}(c, d)$ has four translation classes of vertices, represented by

$$
\left\{\begin{array}{l}
(0,0,0) \\
(c,-c, d), \quad \text { and its cyclic shifts. }
\end{array}\right.
$$

Recall from Section 6 of [24] that the polyhedron $Q(c, d)$, from which $P_{2}(c, d)$ is obtained by the facetting operation, has vertices of multiplicity 2 (every point in $\mathbb{E}^{3}$ taken by a vertex is occupied by exactly two vertices), and thus is not a faithful realization of the underlying abstract polyhedron, if $c$ and $d$ are integers with $c$ odd and $d \equiv 2(\bmod 4)$; for other integral parameters it has single vertices. This explains why the number of translation classes of vertices of $P_{2}(c, d)$ collapses to four in certain cases. In fact, up to similarity, the polyhedra with four translation classes are all of the form $P_{2}(1, k)$ with $k \in \mathbb{Z}$ and $k \equiv 2(\bmod 4)$, so the corresponding polyhedron $Q(1, k)$ does have vertices of multiplicity 2. However, there are also cases with $c$ odd and $d \equiv 2(\bmod 4)$ where $P_{2}(c, d)$ still has eight translation classes of vertices. This happens because $P_{2}(c, d)$ has fewer translational symmetries than $Q(c, d)$; in fact, the translation group of $Q(c, d)$ is $2 \Lambda_{(1,1,0)}$.

We now determine the vertex-stars of $P_{2}(c, d)$. First observe that, since $G=G_{2}(c, d)$ acts transitively on the vertices of $P_{2}(c, d)$, the vertex-stars of $P_{2}(c, d)$ are images of the vertex-star $W_{0}$ at $o$ under the special group $G_{0}\left(\cong S_{4}\right)$, so $G_{0}$ certainly acts transitively on them. The stabilizer of $W_{0}$ contains $\left\langle S_{2}\right\rangle$, and hence the number of vertex-stars cannot exceed eight. This is consistent with the numbers eight or four of transitivity classes of vertices; clearly, vertices in the same translation class must have the same vertex-star. 
The vertex-stars at the eight vertices occurring in Lemma 5.3 are given by

$$
\begin{aligned}
& W_{0}:=\{(c,-c, d),(-c, d, c),(d, c,-c)\}, \\
& W_{1}:=W_{0} U_{1}^{\prime}=\{(-c,-c,-d),(c, d,-c),(-d, c, c)\}, \\
& W_{2}:=W_{0} U_{2}^{\prime}=\{(-c, c, d),(c,-d, c),(-d,-c,-c)\}, \\
& W_{3}:=W_{0} U_{3}^{\prime}=\{(c, c,-d),(-c,-d,-c),(d,-c, c)\}, \\
& W_{4}:=W_{0} T_{1}^{\prime}=\{(-c,-c, d),(c,-d,-c),(d, c, c)\}, \\
& W_{5}:=W_{0} U_{1}^{\prime} T_{1}^{\prime}=\{(c,-c,-d),(-c,-d, c),(-d, c,-c)\}, \\
& W_{6}:=W_{0} U_{2}^{\prime} T_{1}^{\prime}=\{(-c, c,-d),(c, d, c),(d,-c,-c)\}, \\
& W_{7}:=W_{0} U_{3}^{\prime} T_{1}^{\prime}=\{(c, c, d),(-c, d,-c),(-d,-c, c)\},
\end{aligned}
$$

where the labelling accords with the order in which the vertices are listed in (5.14) and (5.15); that is, $W_{0}, \ldots, W_{3}$ for the vertices in (5.14), and $W_{4}, \ldots, W_{7}$ for the vertices in (5.15), in each case with the orders matching.

Each vertex-star consists of three of six vectors in a vertex-star of the corresponding polyhedron $Q(c, d)$. (In the notation of Section 6 of [24], these vertex-stars are $W_{0}, W_{7}$, $W_{6}, W_{5}, W_{1}, W_{4}, W_{2}$ and $W_{3}$, respectively, so in particular the present labelling does not accord with the old one. The reflection $R$ in (6.8) of [24] is not an element of $G$, so it cannot be employed here.) The vertex-stars at the vertices $(c,-c, d),(-c, d, c)$, $(d, c,-c)$ adjacent to $o$ are $W_{6}, W_{4}, W_{7}$, respectively.

Just as in similar situations before, the union of all vertex-stars of $P_{2}(c, d)$ comprises the set of (generally 24 ) vectors

$$
W:=\bigcup_{i=0}^{7} W_{i}=\{( \pm c, \pm c, \pm d),( \pm c, \pm d, \pm c),( \pm d, \pm c, \pm c)\}
$$

which is the same as the corresponding set for $Q(c, d)$ (see (6.13) of [24]). This is also the orbit of $v$ under $G_{0}$. Note that $W$ is the vertex-set of one of the following convex polyhedra: a suitably truncated octahedron or cube, with 24 vertices, if $0<|c|<|d|$ or $0<|d|<|c|$, respectively; a cube if $c= \pm d$; an octahedron if $c=0$; or a cuboctahedron if $d=0$. Figure 5.1 shows the vertex-set of the truncated octahedron obtained for $c=1$ and $d=4$; the fat lines indicate the square faces.

The edge-module of $P_{2}(c, d)$ is given by $\Lambda:=\mathbb{Z}[W]$, the $\mathbb{Z}$-module spanned by the vectors in $W$. As before, $V\left(P_{2}(c, d)\right) \subset \Lambda$. Recall from Lemma 6.2 of [24] that $\Lambda$ is a lattice if and only if $c, d$ can be scaled to a pair of relatively prime integers. Hence we observe that, while the vertex-set of $P_{2}(c, d)$ is always discrete, its edge-module is generally not discrete. On the other hand, the vertex-set of $Q(c, d)$ is discrete if and only if its edge-module is discrete; in fact, in this case, $2 \Lambda$ is a subset of the vertex-set.

The eight vertex-stars in (5.16) are mutually disjoint (and hence distinct), unless $c= \pm d$ or $c=0$ or $d=0$; the circled points in Fig. 5.1 indicate the vertex-star $W_{0}$ for $c=1$ and $d=4$. The vertex-stars are still mutually distinct (but not disjoint) if $c= \pm d$ or $c=0$; in these cases they correspond to the triangular faces of a stella octangula (a pair of regular tetrahedra inscribed in a cube as in Fig. 3.1) or an octahedron, respectively. Finally, if $d=0$, the vertex-stars coincide in pairs, namely we have

$$
W_{0}=W_{5}, \quad W_{1}=W_{4}, \quad W_{2}=W_{6}, \quad W_{3}=W_{7} ;
$$

now the vertex-stars consist of alternating vertices of the four equatorial hexagons in the cuboctahedron. 


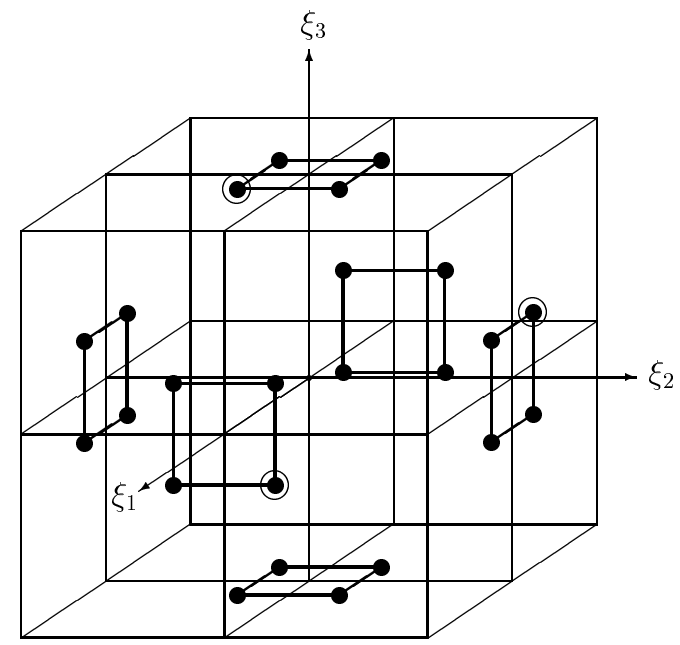

Fig. 5.1. The set $W$ for $c=1$ and $d=4$. The points in the vertex-star $W_{0}$ for $P_{2}(c, d)$ are circled. The cubes are drawn in for reference.

We now have

Lemma 5.5. $\quad P_{2}(c, d)$ is a geometric polyhedron, except when $d=k c$ and $k$ is an integer with $k \equiv 2(\bmod 4)$.

Proof. The case $c=0$ is obvious. Suppose $c \neq 0$. We have altogether eight translation classes of vertices and eight vertex-stars, unless $d=0$ or $d=k c$ with $k \in \mathbb{Z}$ and $k \equiv$ $2(\bmod 4)$. Moreover, the vertex-stars at vertices of distinct translation classes are also distinct, so each translation class of vertices is characterized by its unique vertex-star. Hence $P_{2}(c, d)$ cannot have multiple vertices. To see this more explicitly, let $x$ be a vertex of $P_{2}(c, d)$ with vertex-star $W_{j}$ (say), and let $y$ be a vertex adjacent to $x$ such that $y-x \in W_{j}$. We must prove that $x-y$ belongs to the vertex-star $W_{k}$ (say) at $y$, that is, $x \in y+W_{k}$. Reducing $x$ modulo $4 c \mathbb{Z}^{3}$ (if need be), we may further assume that $x$ is among the eight vertices listed in Lemma 5.3. Now inspection of the corresponding adjacent vertices and their vertex-stars shows that the required property holds in each case.

If $d=k c$ with $k \in \mathbb{Z}$ and $k \equiv 2(\bmod 4)$, then we have four translation classes of vertices but still eight vertex-stars. Hence each point occupied by a vertex is occupied by two vertices, so we have a double vertex associated with two (disjoint) vertex-stars; then this point has "valency" 6 . Finally, when $d=0$, there are eight translation classes of vertices and four vertex-stars, so each vertex-star is associated with two translation classes. Now we have a regular geometric polyhedron (see (5.6)).

The vertex-stars of $P_{2}(c, d)$ are generally not planar, although the vertex-figures are triangles. In fact, we have

Lemma 5.6. The vertex-stars of $P_{2}(c, d)$ are planar if and only if $d=0$. 
Proof. The three vectors in $W_{0}$ have determinant $\pm d\left(3 c^{2}+d^{2}\right)$, so they lie in a plane if and only if $d=0$.

Next we determine the regular polyhedra.

Lemma 5.7. The polyhedron $P_{2}(c, d)$ is geometrically chiral if $c, d \neq 0$, or geometrically regular if $c=0$ or $d=0$. In particular, $P_{2}(c, 0)$ is similar to $P_{2}(1,0)=\{\infty, 3\}^{(b)}$, and $P_{2}(0, d)$ is similar to $P_{2}(0,1)=\{4,3\}$.

Proof. The second claim was already settled (see (5.6) and (5.7)). Now suppose $c, d \neq$ 0 . As in the proof of Lemma 4.6 we must refute the assumption that there exists an involutory symmetry $R$ which fixes $o$ and $v$ and interchanges the neighbors $(-c, d, c)$ and $(d, c,-c)$ of $o$. Since $W_{0}$ is not planar when $d \neq 0$, this symmetry $R$ must necessarily be the reflection in the plane through $o, v$ with normal vector

$$
n:=(-c, d, c)-(d, c,-c)=(-c-d,-c+d, 2 c) .
$$

Moreover, since $R^{-1} T R=T$, the rotation axis of $T$, with direction vector $e_{1}+e_{2}$, must necessarily be invariant under $R$. However, $e_{1}+e_{2}$ is not a scalar multiple of $n$, so $e_{1}+e_{2}$ must lie in the mirror of $R$. Hence $-2 c=n \cdot\left(e_{1}+e_{2}\right)=0$, which is a contradiction.

The discussion of enantiomorphism is similar to that in the previous section. Now we have

$$
P_{2}(-c, d)=P_{2}(c, d) R,
$$

where $R$ is the reflection (in the plane $\xi_{1}=\xi_{2}$ ) given by

$$
R:\left(\xi_{1}, \xi_{2}, \xi_{3}\right) \mapsto\left(\xi_{2}, \xi_{1}, \xi_{3}\right) .
$$

In this case conjugation by $R$ transforms the pair of generators $T(c, d), S_{2}$ for $G_{2}(c, d)$ to the pair of generators $T(-c, d), S_{2}^{-1}$ for $G_{2}(-c, d)$, so in particular we have $G_{2}(-c, d)=$ $R^{-1} G_{2}(c, d) R$. However, $R \notin G_{2}(c, d)$, because $G_{2}(c, d)$ consists only of direct isometries. As before, if $\Phi(c, d):=\left\{F_{0}(c, d), F_{1}(c, d), F_{2}(c, d)\right\}$ is the base flag of $P_{2}(c, d)$, and if $\Phi^{2}(c, d):=\left\{F_{0}(c, d), F_{1}(c, d), F_{2}^{\prime}(c, d)\right\}$ denotes the adjacent flag differing from $\Phi(c, d)$ in its 2-face, then we can use arguments for $G_{2}(c, d)$ similar to those in (4.19), (4.20) and (4.21) for $G_{1}(a, b)$ to establish (5.19) as well as

$$
\Phi(-c, d) R=\Phi^{2}(c, d), \quad \Phi^{2}(-c, d) R=\Phi(c, d) .
$$

Here the equations take the form

$$
\begin{aligned}
& V\left(P_{2}(-c, d)\right)=o G_{2}(-c, d)=o R^{-1} G_{2}(c, d) R=o G_{2}(c, d) R=V\left(P_{2}(c, d)\right) R, \\
& F_{1}(-c, d) G_{2}(-c, d)=F_{1}(-c, d) R^{-1} G_{2}(c, d) R=\left(F_{1}(c, d) G_{2}(c, d)\right) R, \\
& F_{2}(-c, d) G_{2}(-c, d)=F_{2}(-c, d) R^{-1} G_{2}(c, d) R=\left(F_{2}(c, d)^{\prime} G_{2}(c, d)\right) R .
\end{aligned}
$$

One implication is again that Wythoff's construction applied to $G_{2}(c, d)$ with the alternative pair of generators $T(c, d), S_{2}^{-1}$ and the same initial vertex, yields the same polyhedron $P_{2}(c, d)$ as before, but now with a different base flag, namely $\Phi^{2}(c, d)$. 
Alternatively we could have appealed to analogous results for the polyhedra $Q(c, d)$, again observing that conjugation by $R$ commutes with the facetting operation $\varphi_{2}$ (see Section 6 of [24]); in particular,

$$
(Q(c, d) R)^{\varphi_{2}}=\left(Q(c, d)^{\varphi_{2}}\right) R .
$$

We can also tell when two polyhedra are affinely equivalent. The result is consistent with Lemma 6.10 of [24] for the polyhedra $Q(c, d)$.

Lemma 5.8. Let $c, d, e, f$ be real numbers, and let $(c, d) \neq(0,0) \neq(e, f)$. Then the polyhedra $P_{2}(c, d)$ and $P_{2}(e, f)$ are affinely equivalent if and only if $(e, f)=s( \pm c, d)$ for some non-zero scalar s. Moreover, $P_{2}(c, d)$ and $P_{2}(e, f)$ are congruent if and only if $(e, f)=( \pm c, \pm d)$.

Proof. Suppose we have an affine transformation $S$ with $P_{2}(e, f)=P_{2}(c, d) S$. As in the proof of Lemma 4.7 we can assume that $o S=o$ and $(c,-c, d) S=(e,-e, f)$. First note that, since $S$ and $S^{-1}$ preserve planarity of vertex-stars, we certainly have $d=0$ if and only if $f=0$, so in this case we are done. Now let $d \neq 0$, so that the vertex-stars are not planar. Then we can argue as in Lemma 4.7 and conclude that $S^{-1} T(c, d) S=T(e, f)$ and $S^{-1} S_{2} S=S_{2}$ or $S_{2}^{-1}$. In particular, we must have $S=s I$ or $S=s R$, with $R$ as in (5.20), for some scalar $s$. It follows that $(e, f)=s( \pm c, d)$. Moreover, if $S$ is an isometry, then $s= \pm 1$. The converse is obvious (by (5.19)).

The affine equivalence classes of polyhedra $P_{2}(c, d)$ are illustrated in Fig. 5.2. At the corners of the square we find the regular polyhedra $P_{2}(1,0)=\{\infty, 3\}^{(b)}$ and $P_{2}(0,1)=$ $\{4,3\}$. Each class is represented by one polyhedron whose parameter point $(c, d)$ is located on the upper right side of the square; the other three sides of the square give alternative representations, as indicated by the four symmetrically related nodes.

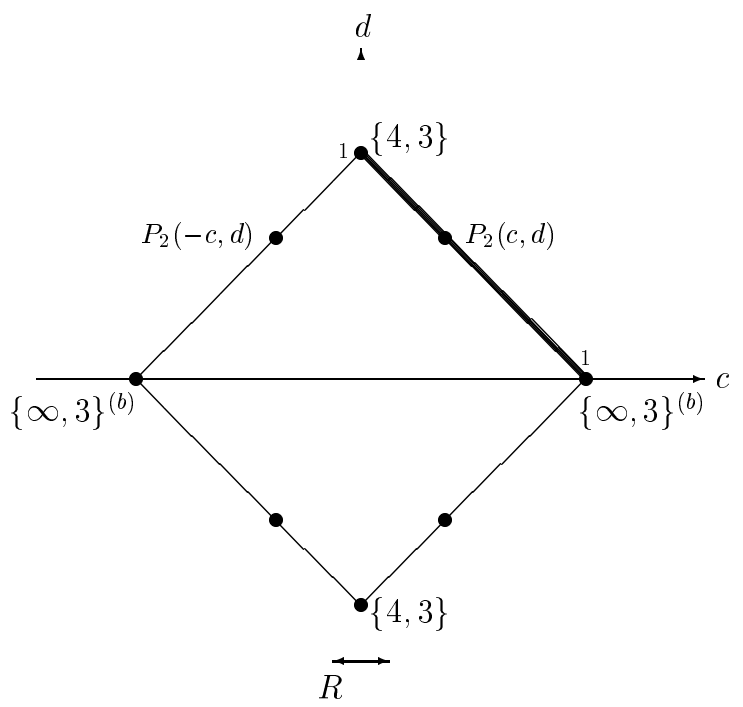

Fig. 5.2. The affine classes of polyhedra $P_{2}(c, d)$. 
As $G_{2}(c, d)$ contains only direct isometries, the faces of $P_{2}(c, d)$ consist of either all right-hand helices or all left-hand helices. Each face has its axis parallel to a coordinate axis. The reflection $R$ of (5.20) changes orientation, so the faces of $P_{2}(c, d)$ are righthand helices if and only if the faces of $P_{2}(-c, d)$ are left-hand helices. This is similar to what we observed in the previous section. The following lemma describes the translation classes of faces modulo $4 c \mathbb{Z}^{3}$; in the regular case they coincide with the translation classes relative to the full symmetry group. There are six classes, even when there are only four translation classes of vertices.

Lemma 5.9. $\quad P_{2}(c, d)$ has precisely six translation classes of faces, namely, those represented by the faces $F_{2}, F_{2} T_{1}, F_{2} S_{2}, F_{2} T_{1} S_{2}, F_{2} S_{2}^{2}$ and $F_{2} T_{1} S_{2}^{2}$, where $F_{2}$ is the base face given by (5.4).

Proof. The details are tedious, so we just outline the argument. Suppose $c \neq 0$. Each vertex of $P_{2}(c, d)$ is equivalent modulo $4 c \mathbb{Z}^{3}$ to one of the eight vertices $o, o U_{1}, o U_{2}$, $o U_{3}, o T_{1}, o U_{1} T_{1}, o U_{2} T_{1}$ or $o U_{3} T_{1}$ (see the proof of Lemma 5.3), so each face is equivalent to a face containing one of these vertices. This latter set of faces consists of the three faces $F_{2}, F_{2} S_{2}, F_{2} S_{2}^{2}$ containing $o$, as well as their images under $U_{1}, U_{2}, U_{3}, T_{1}, U_{1} T_{1}$, $U_{2} T_{1}$ and $U_{3} T_{1}$. Inspection of these faces then reduces the number of classes to six, with two classes for each possible direction of axes. The representative faces include $F_{2}$ and $F_{2} T_{1}$, which are parallel to the $y$-axis, as well as their images under $S_{2}$ and $S_{2}^{2}$ (see also (5.12)). The six faces are those depicted in Fig. 4.3, with $F_{2} T_{1}=F_{2} S_{2}^{2} T S_{2}$.

\section{Type $\{\infty, 4\}$, with Helical Faces over Triangles}

In this section we describe the third family of chiral apeirohedra with helical faces. In this case the polyhedra are of type $\{\infty, 4\}$ and have faces consisting of helices over triangles. Again we employ Wythoff's construction.

Now we must begin with an irreducible group $G=\left\langle S_{1}, S_{2}\right\rangle$ with special group $G_{0}=[3,4]^{+}$, where $S_{1}^{\prime}, S_{2}$ are standard generators of $G_{0}$ considered as the group of the octahedron $\{3,4\}$, so that $S_{1}^{\prime}$ and $S_{2}$ have periods 3 and 4 , respectively, and their product has period 2. As in similar situations discussed before, once $S_{2}$ has been selected, there are four equivalent choices for $S_{1}^{\prime}$ such that $T^{\prime}=S_{1}^{\prime} S_{2}$ has period 2; if $S_{1}^{\prime}$ is one of them, then the other three are $S_{2}^{-1} S_{1}^{\prime} S_{2}, S_{2}^{-2} S_{1}^{\prime} S_{2}^{2}$ and $S_{2}^{-3} S_{1}^{\prime} S_{2}^{3}$. Moreover, as before, replacing $S_{2}$ by its inverse would give the same polyhedron.

Again we consider specific representations for the generators. Now we take

$$
\begin{array}{ll}
S_{1}^{\prime}: \quad x & \left.\mapsto \xi_{3}, \xi_{1}, \xi_{2}\right), \\
S_{2}: \quad x & \left.\mapsto \xi_{3}, \xi_{2},-\xi_{1}\right)
\end{array}
$$

as generators of $G_{0}$; these are the inverses of the elements $S_{2}$ and $S_{1}^{\prime}$ of (5.1). Then $T^{\prime}$ and $T$ are the same transformations as in the previous section. However, the resulting group $G=G_{3}(c, d)$ is not the same as $G_{2}(c, d)$; in fact, the two groups have different translation subgroups. In particular, $G_{3}(c, d)$ is generated by

$$
\begin{aligned}
& S_{2}: x \mapsto\left(\xi_{3}, \xi_{2},-\xi_{1}\right), \\
& T: \quad x \mapsto\left(\xi_{2}, \xi_{1},-\xi_{3}\right)+(c,-c, d),
\end{aligned}
$$


with real parameters $c$ and $d$, not both zero. Then

$$
\begin{aligned}
& S_{1}: \quad x \quad\left(\xi_{3}, \xi_{1}, \xi_{2}\right)+(-d,-c, c), \\
& S_{1}^{3}: \quad x \mapsto\left(\xi_{1}, \xi_{2}, \xi_{3}\right)+d(-1,-1,-1) .
\end{aligned}
$$

Then $S_{1}^{3}$ is the translation by $d(-1,-1,-1)$, and is trivial if $d=0$. The special case $d=0$ gives the finite group

$$
G_{3}(c, 0) \cong[3,4]^{+}
$$

(with fixed point $(0,-c, 0)$ ); the polyhedron is an octahedron.

The polyhedron $P=P_{3}(c, d)$ is obtained from $G=G_{3}(c, d)$ by Wythoff's construction with base vertex $F_{0}=o$. Its base edge $F_{1}$ has vertices $o$ and $v:=o T=(c,-c, d)$, and hence is the same as for $P_{2}(c, d)$. The base face $F_{2}$ is given by

$$
F_{2}=o\left\langle S_{1}\right\rangle=\{(-d,-c, c),(0,0,0),(c,-c, d)\}+\mathbb{Z} \cdot d(1,1,1)
$$

and consists of a helix over a triangle. All other vertices, edges and faces of $P$ are the images of $F_{0}, F_{1}$ and $F_{2}$ under $G$. The vertices adjacent to $o$ are

$$
v=(c,-c, d), \quad v S_{2}=(d,-c,-c), \quad v S_{2}^{2}=(-c,-c,-d), \quad v S_{2}^{3}=(-d,-c, c) .
$$

The polyhedron $P_{3}(c, d)$ is similar to $P_{3}(1, d / c)$ or $P_{3}(0,1)$ according as $c \neq 0$ or $c=0$, so again there is only a single parameter for the similarity classes.

In Section 8 (and Section 7) we establish a link between $P_{3}(c, d)$ and the finite-faced polyhedron $Q(-d, 2 c)$ (note the change of parameters). However, the facetting operation $\varphi_{2}$ does not apply in this context.

Next we find the translation subgroup $T(G)$ of $G=G_{3}(c, d)$. Since $S_{1}^{3}$ is the translation by $d(-1,-1,-1)$, and its conjugates under the special group $G_{0}\left(\cong[3,4]^{+}\right)$ consists of the vectors $d( \pm 1, \pm 1, \pm 1)$, we see that

$$
d \Lambda_{(1,1,1)} \leqslant T(G)
$$

In fact, we have

Lemma 6.1. The translation subgroup of $G_{3}(c, d)$ is given by $T\left(G_{3}(c, d)\right)=d \Lambda_{(1,1,1)}$.

Proof. Now $G=N \cdot\left\langle S_{2}\right\rangle$, where $N:=\left\langle T_{0}, T_{1}, T_{2}, T_{3}\right\rangle$ with $T_{j}:=S_{2}^{-j} T S_{2}^{j}$ for $j=0,1,2,3$. (The labelling of the half-turns $T_{j}$ differs from that in previous sections.) In particular,

$$
\begin{aligned}
& T_{0}=T: \quad x \mapsto\left(\xi_{2}, \xi_{1},-\xi_{3}\right)+(c,-c, d), \\
& T_{1}: x \mapsto\left(-\xi_{1},-\xi_{3},-\xi_{2}\right)+(d,-c,-c), \\
& T_{2}: x \mapsto\left(-\xi_{2},-\xi_{1},-\xi_{3}\right)+(-c,-c,-d), \\
& T_{3}: \quad x \mapsto\left(-\xi_{1}, \xi_{3}, \xi_{2}\right)+(-d,-c, c) .
\end{aligned}
$$

Note that $N$ is normal in $G$. 
The element $T_{0} T_{1} T_{2} T_{3} T_{2} T_{1}$ of $N$ is the translation by $(0,0,-2 d)$. Its conjugates by $S_{1}$ and $S_{1}^{2}$ are also in $N$ and are the translations by $(-2 d, 0,0)$ and $(0,-2 d, 0)$, respectively. Hence $2 d \mathbb{Z}^{3} \leqslant N$; in fact, $2 d \mathbb{Z}^{3}$ is normal in $N$. Moreover, $S_{2}^{2} T_{0} T_{2} T_{1} T_{3}$ is the translation by $(-2 d, 0,-2 d)$ contained in $N$, so $S_{2}^{2} \in N$ and $N$ has index at most 2 in $G$.

Now consider the quotient $M:=N / 2 d \mathbb{Z}^{3}$. The elements $\left(T_{j} T_{j+1}\right)^{3}$ with $j=0,1,2,3$ (considered modulo 4), as well as $\left(T_{0} T_{2}\right)^{2}$, are translations in $2 d \mathbb{Z}^{3}$, hence each is trivial when considered in $M$. Moreover, since $T_{0} T_{1} T_{2} T_{3} T_{2} T_{1}$ is also in $2 d \mathbb{Z}^{3}$, we have $T_{3}=$ $T_{2} T_{1} T_{0} T_{1} T_{2}$ in $M$, so $M$ is generated by (the images of) $T_{0}, T_{1}, T_{2}$. These generators

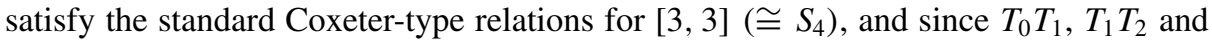
$T_{0} T_{2}$ themselves are not translations, these relations give a presentation for $M$, so in particular $M \cong S_{4}$. On the other hand, the normal subgroup $d \Lambda_{(1,1,1)}$ of $G$ (contained in $T(G)$ ) is not a (normal) subgroup of $N$; otherwise, since $2 d \mathbb{Z}^{3}$ has index 2 in $d \Lambda_{(1,1,1)}$, the group $N / d \Lambda_{(1,1,1)}$ would be a quotient of $N / 2 d \mathbb{Z}^{3}$ of order 12 (however, $S_{4}$ does not have such a quotient). Hence $N$ must be a proper subgroup of $G$ (that is, $S_{2} \notin N$ ), and $G=N \cdot T(G)$. Now it follows that

$$
N / 2 d \mathbb{Z}^{3}=M \cong S_{4} \cong[3,4]^{+} \cong G_{0} \cong G / T(G) \cong N /(T(G) \cap N),
$$

so in particular $T(G) \cap N=2 d \mathbb{Z}^{3}$ and $2 d \mathbb{Z}^{3}$ has index 2 in $T(G)$. Hence $T(G)=$ $d \Lambda_{(1,1,1)}$, as claimed.

Again we observe that the translation group depends only on a single parameter, $d$ in this case. Now Lemma 6.1 can be rephrased as

$$
G_{3}(c, d) / d \Lambda_{(1,1,1)} \cong[3,4]^{+} \cong S_{4},
$$

where each term represents the special group.

The next lemma describes the vertex-set of $P_{3}(c, d)$, which again is discrete for all parameter values.

Lemma 6.2. The vertex-set of $P_{3}(c, d)$ is given by

$$
\begin{aligned}
V\left(P_{3}(c, d)\right)= & \{(0,0,0),(c,-c, d),(-c,-c, d),(0,-2 c, 0),(d,-c, c),(d,-c,-c)\} \\
& +d \Lambda_{(1,1,1)} .
\end{aligned}
$$

The six cosets of $d \Lambda_{(1,1,1)}$ occurring are distinct, except when $c / d$ is an integer. If $c / d$ is an odd integer, then $V\left(P_{3}(c, d)\right)=d \Lambda_{(1,1,1)}$ (and the cosets all coincide). If $c / d$ is an even integer, then

$$
V\left(P_{3}(c, d)\right)=\{(0,0,0),(c,-c, d),(d,-c, c)\}+d \Lambda_{(1,1,1)}
$$

(and the cosets coincide in pairs).

Proof. We appeal to the proof of Lemma 6.1. First observe that, modulo $d \Lambda_{(1,1,1)}$, each element of $G$ can be written as

$$
S_{2}^{i}\left(T_{0} T_{1} T_{2}\right)^{j}\left(T_{0} T_{1}\right)^{k} T_{0}^{m}
$$


with $i=0,1, j=0,1,2,3, k=0,1,2$ and $m=0,1$. In fact, the two elements $I, S_{2}$ give a system of left coset representatives of $G$ modulo its subgroup $N$, and the four elements $\left(T_{0} T_{1} T_{2}\right)^{j}$ with $j=0,1,2,3$ give a system of left coset representatives of

$$
N / 2 d \mathbb{Z}^{3}=M=\left\langle T_{0}, T_{1}, T_{2}\right\rangle \cong[3,3]
$$

modulo its subgroup $\left\langle T_{0}, T_{1}\right\rangle\left(\cong S_{3}\right)$; moreover, since $2 d \mathbb{Z}^{3} \leqslant d \Lambda_{(1,1,1)}$, representations of elements modulo $2 d \mathbb{Z}^{3}$ also yield representations modulo $d \Lambda_{(1,1,1)}$.

The vertex-set of $P_{3}(c, d)$ is the orbit of $o$ under $G$ and consists of cosets of $d \Lambda_{(1,1,1)}$, namely those of the images of $o$ under the elements in (6.7). The first term in (6.7) fixes $o$, because $S_{2}$ fixes $o$. Next, it is easily verified that $T_{0} T_{1} T_{2}=S_{2}^{-1}$ in $G / d \Lambda_{(1,1,1)}$, so the second term of (6.7) also does not contribute a non-trivial coset. Thus contributions can only arise from the images of $o$ under the six elements

$$
\left(T_{0} T_{1}\right)^{k} T_{0}^{m}
$$

with $k=0,1,2$ and $m=0,1$. Modulo $d \Lambda_{(1,1,1)}$, these images are $(0,0,0),(-c,-c, d)$, $(d,-c, c)$ if $k=0,1,2$ and $m=0$, and $(c,-c, d),(0,-2 c, 0),(d,-c,-c)$ if $k=$ $0,1,2$ and $m=1$. They give a set of six vertices modulo $d \Lambda_{(1,1,1)}$.

The corresponding six cosets are distinct, unless two representing vertices become equivalent modulo $d \Lambda_{(1,1,1)}$. Inspection shows that such equivalencies occur precisely when $c / d \in \mathbb{Z}$. If $c / d$ is an odd integer, then the cosets all coincide and hence $V\left(P_{3}(c, d)\right)$ $=d \Lambda_{(1,1,1)}$. However, if $c / d$ is an even integer, then there are three distinct cosets represented by $(0,0,0),(c,-c, d)$ or $(d,-c, c)$. This completes the proof.

In the exceptional cases of Lemma 6.2, with $c \neq 0$, the polyhedra are not faithful realizations of the underlying abstract polyhedra. In fact, we prove in Lemma 6.3 that $P_{3}(c, d)$ is a geometric polyhedron, except when $c / d$ is a non-zero integer. When $c=0$, we obtain a (faithful) regular polyhedron (see Lemma 6.5). Moreover, note that, when $d=0$, the polyhedron is the (finite) octahedron $P_{3}(c, 0)$ whose vertices are the six points listed in Lemma 6.2.

Next we determine the vertex-stars of $P_{3}(c, d)$. They are the images of the vertex-star $W_{0}$ at $o$ under the special group $G_{0}\left(\cong S_{4}\right)$, so the latter certainly acts transitively on them. On the other hand, since $W_{0}$ is stabilized by $\left\langle S_{2}\right\rangle$, the number of vertex-stars cannot exceed six. This bound is consistent with the numbers six, three or one of transitivity classes of vertices.

The vertex-stars at the six representing vertices of Lemma 6.2 are given by

$$
\begin{aligned}
W_{0}:= & \{(c,-c, d),(d,-c,-c),(-c,-c,-d),(-d,-c, c)\}, \\
W_{1}:=W_{0} T_{0}^{\prime}= & \{(-c, c,-d),(-c, d, c),(-c,-c, d),(-c,-d,-c)\}, \\
W_{2}:=W_{0} T_{0}^{\prime} T_{1}^{\prime}= & \{(c, c, d),(c, d,-c),(c,-c,-d),(c,-d, c)\}, \\
W_{3}:=W_{0} T_{0}^{\prime} T_{1}^{\prime} T_{0}^{\prime}= & \{(d, c, c),(-c, c, d),(-d, c,-c),(c, c,-d)\}, \\
W_{4}:=W_{0} T_{1}^{\prime} T_{0}^{\prime}= & \{(d, c,-c),(-c, d,-c),(-d,-c,-c),(c,-d,-c)\}, \\
W_{5}:=W_{0} T_{1}^{\prime}= & \{(-d, c, c),(c, d, c),(d,-c, c),(-c,-d, c)\}
\end{aligned}
$$

(see (6.8)), where the labelling matches the order in which the vertices are listed in the lemma. Note here that $\left(T_{0}^{\prime} T_{1}^{\prime}\right)^{3}=I$. The vertex-stars at the vertices $(c,-c, d)$, 


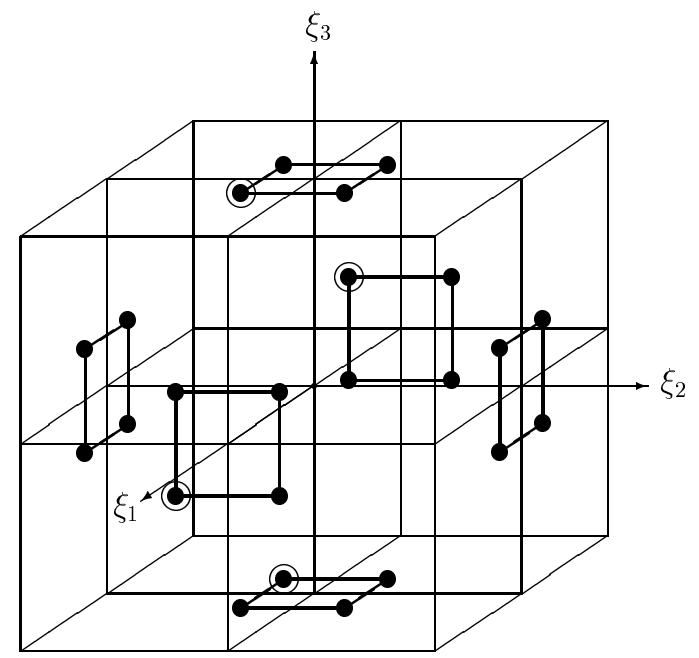

Fig. 6.1. The set $W$ for $c=1$ and $d=4$. Now the points in the vertex-star $W_{0}$ for $P_{3}(c, d)$ are circled.

$(d,-c,-c),(-c,-c,-d)$ and $(-d,-c, c)$ adjacent to $o$ are $W_{1}, W_{5}, W_{2}$ and $W_{4}$, respectively. The vertex-star at an arbitrary vertex $x$ is found by first reducing $x$ modulo $d \Lambda_{(1,1,1)}$ to a representing vertex and then taking the vertex-star at that vertex.

The union $W$ of the vertex-stars of $P_{3}(c, d)$ is the same set as in the previous section, that is,

$$
W:=\bigcup_{i=0}^{5} W_{i}=\{( \pm c, \pm c, \pm d),( \pm c, \pm d, \pm c),( \pm d, \pm c, \pm c)\}
$$

Hence the edge-module $\Lambda:=\mathbb{Z}[W]$ of $P_{3}(c, d)$ is also the same as before.

The six vertex-stars in (6.9) are mutually disjoint (and hence distinct), unless $c= \pm d$ or $c=0$ or $d=0$; Fig. 6.1 illustrates the vertex-star $W_{0}$. The vertex-stars are still mutually distinct (but not disjoint) if $c= \pm d$ or $d=0$; in these cases they correspond to the square faces of a cube or cuboctahedron, respectively. Finally, if $c=0$, the vertex-stars coincide in pairs and consist of the equatorial squares of an octahedron; in particular,

$$
W_{0}=W_{3}, \quad W_{1}=W_{2}, \quad W_{4}=W_{5}
$$

Next we prove

Lemma 6.3. $\quad P_{3}(c, d)$ is a geometric polyhedron, except when $c / d$ is a non-zero integer.

Proof. The case $d=0$ is trivial, so let $d \neq 0$. Then we have six translation classes of vertices and six vertex-stars, except when $c / d$ is an integer (possibly zero). Moreover, the vertex-stars at vertices of distinct translation classes are distinct, so again each translation class of vertices is characterized by its unique vertex-star. Hence $P_{3}(c, d)$ cannot have multiple vertices. Again we can see this more explicitly. Let $x$ be a vertex of $P_{3}(c, d)$ with vertex-star $W_{j}$, and let $y$ be a vertex adjacent to $x$ such that $y-x \in W_{j}$. We must 
verify that $x-y$ belongs to the vertex-star $W_{k}$ at $y$, that is, $x \in y+W_{k}$. Reducing $x$ modulo $d \Lambda_{(1,1,1)}$ we can achieve that $x$ is among the six vertices listed in Lemma 6.2. Inspection of the corresponding adjacent vertices and their vertex-stars now establishes the required property in each case.

If $c / d$ is a non-zero even integer, then we have three translation classes of vertices and six vertex-stars, so again each vertex has multiplicity 2 and is associated with two disjoint vertex-stars.

If $c / d$ is an odd integer, then there is just one translation class of vertices but still six vertex-stars. Now each point $x$ taken by a vertex is in fact occupied by six vertices, so each vertex has multiplicity 6 and all six vertex-stars occur at it. Hence the set of points connected to $x$ by an edge of $P_{3}(c, d)$ is given by $x+W$. This case is highly degenerate.

Finally, when $c=0$, there are three translation classes of vertices and three vertexstars, so we have a geometric polyhedron. As we shall see, the polyhedron is regular.

Since $S_{2}$ is a rotation about the $\xi_{2}$-axis, it is clear that the four vertices of $P_{3}(c, d)$ adjacent to $o$ all lie in a plane perpendicular to the $\xi_{2}$-axis, so the vertex-figures certainly are planar. Moreover, we have

Lemma 6.4. The vertex-stars of $P_{3}(c, d)$ are planar if and only if $c=0$.

Proof. The vertex-stars are planar if and only if the affine hull of the vertex-figure at $o$ contains $o$ itself. This occurs if and only if $v=(c,-c, d)$ lies in the $\xi_{1} \xi_{3}$-plane. Hence the condition is $c=0$.

Next we determine the regular polyhedra. Recall from pp. 224 and 230 of [20] that $\{\infty, 4\}_{, * 3}$ denotes the (self-Petrie) regular polyhedron of type $\{\infty, 4\}$, whose full symmetry group is specified by the single extra relation

$$
\left(R_{2}\left(R_{1} R_{0}\right)^{2}\right)^{3}=I .
$$

(The "." in the suffix means that the length of the Petrie polygons is unspecified, and the 3 with $*$ prefix indicates the length of the 2-zigzags of the combinatorial dual.)

Lemma 6.5. The polyhedron $P_{3}(c, d)$ is geometrically chiral if $c, d \neq 0$, or geometrically regular if $c=0$ ord $=0$. In particular, $P_{3}(0, d)$ is similar to $P_{3}(0,1)=\{\infty, 4\}_{, * 3}$, and $P_{3}(c, 0)$ is similar to $P_{3}(1,0)=\{3,4\}$.

Proof. Clearly, by construction, $P_{3}(c, d)$ is at least chiral. Now suppose $P_{3}(c, d)$ is regular. Then there exists an involutory symmetry $R$ which fixes $o$ and $v\left(\right.$ and $v S_{2}^{2}$ ) and interchanges the vertices $v S_{2}$ and $v S_{2}^{-1}$ in the vertex-figure at $o$; moreover, $R S_{2} R=S_{2}^{-1}$ and $R T R=T$. If $c \neq 0$, the vectors $v=(c,-c, d)$ and $v S_{2}^{2}=(-c,-c,-d)$ span an $R$-invariant plane with normal vector

$$
n:=v S_{2}-v S_{2}^{-1}=(2 d, 0,-2 c),
$$

so $R$ must be the reflection in this plane. The rotation axis of $T$ is the line through $\frac{1}{2} v$ with direction vector $e_{1}+e_{2}$. (As before, $e_{1}, e_{2}, e_{3}$ denotes the standard basis of $\mathbb{E}^{3}$.) 
Since $R$ must also leave this line invariant, it must lie in the mirror of $R$; in fact, it cannot be perpendicular to the mirror because $e_{1}+e_{2}$ and $n$ are not collinear. However, then $d=n \cdot\left(e_{1}+e_{2}\right)=0$. Hence, if $P_{3}(c, d)$ is regular, then either $c=0$ or $d=0$. The latter case yields the regular octahedron $P_{3}(c, 0)$.

Now let $c=0$. Suppose $R$ is an involutory symmetry of $P_{3}(0, d)$ with the properties mentioned. Then $v=d e_{3}=-v S_{2}^{2}$ and $v S_{2}=d e_{1}=-v S_{2}^{-1}$, so necessarily $e_{3} R=e_{3}$ and $e_{1} R=-e_{1}$. Moreover, since $R S_{2} R=S_{2}^{-1}$, the symmetry $R$ must leave the rotation axis of $S_{2}$ (the $y$-axis) invariant, so we also have $e_{2} R= \pm e_{2}$. We can further eliminate the possibility that $R$ is a plane reflection; in fact, then necessarily $e_{2} R=e_{2}$, so $R$ would not leave the rotation axis of the half-turn $T$ invariant. This, then, leaves only the possibility that $R$ is the half-turn about the $z$-axis (spanned by $v$ ). On the other hand, it is straightforward to check that this half-turn, denoted again by $R$, indeed is a symmetry of $P_{3}(0, d)$ with the required properties. In particular, it fixes $o$ and $v$ and maps the base face $F_{2}$ of (6.4) to the face

$$
\begin{aligned}
F_{2} R & =\{(d, 0,0),(0,0,0),(0,0, d)\}+\mathbb{Z} \cdot d(-1,-1,1) \\
& =\{(0,-d, d),(0,0, d),(0,0,0)\}+\mathbb{Z} \cdot d(1,1,-1)=F_{2} T
\end{aligned}
$$

adjacent to $F_{2}$ along the base edge. It follows that $P_{3}(0, d)$ is regular (see Lemma 2.1). Moreover, since $R_{2}:=R$ and $S_{2}$ are rotations and $S_{1}$ is a twist, the symmetries $R_{1}:=S_{2} R_{2}^{-1}$ and $R_{0}:=S_{1} R_{1}^{-1}$ must also be half-turns. Hence $P_{3}(0, d)$ has dimension vector

$$
\left(\operatorname{dim} R_{0}, \operatorname{dim} R_{1}, \operatorname{dim} R_{2}\right)=(1,1,1) .
$$

Comparison with the regular polyhedra in $\mathbb{E}^{3}$ now establishes that $P_{3}(0, d)$ is similar to $\{\infty, 4\}_{, * 33}$ (see p. 225 of [20]).

The discussion of enantiomorphism follows the same pattern as in previous sections. Now

$$
P_{3}(c,-d)=P_{3}(c, d) R,
$$

where $R$ is the reflection (in the plane $\xi_{3}=0$ ) given by

$$
R:\left(\xi_{1}, \xi_{2}, \xi_{3}\right) \mapsto\left(\xi_{1}, \xi_{2},-\xi_{3}\right) .
$$

In this case conjugation by $R$ transforms the generators $T(c, d), S_{2}$ for $G_{3}(c, d)$ to the generators $T(c,-d), S_{2}^{-1}$ for $G_{3}(c,-d)$, so in particular $G_{3}(c,-d)=R^{-1} G_{3}(c, d) R$. However, $R \notin G_{3}(c, d)$, again because $G_{3}(c, d)$ only contains direct isometries. If $\Phi(c, d):=\left\{F_{0}(c, d), F_{1}(c, d), F_{2}(c, d)\right\}$ is the base flag of $P_{3}(c, d)$, and if

$$
\Phi^{2}(c, d):=\left\{F_{0}(c, d), F_{1}(c, d), F_{2}^{\prime}(c, d)\right\}
$$

is the adjacent flag differing from $\Phi(c, d)$ in its 2-face, then equations for $G_{3}(c, d)$ similar to those in (5.21) for $G_{2}(c, d)$ establish (6.12) and

$$
\Phi(c,-d) R=\Phi^{2}(c, d), \quad \Phi^{2}(c,-d) R=\Phi(c, d) .
$$

In particular, this implies that Wythoff's construction applied to $G_{3}(c, d)$ with the alternative pair of generators $T(c, d), S_{2}^{-1}$ and the same initial vertex, yields the same polyhedron $P_{3}(c, d)$ as before, but now with $\Phi^{2}(c, d)$ as the base flag. 
Next we investigate affine equivalence. Here we have

Lemma 6.6. Let $c, d, e, f$ be real numbers, and let $(c, d) \neq(0,0) \neq(e, f)$. Then the polyhedra $P_{3}(c, d)$ and $P_{3}(e, f)$ are affinely equivalent if and only if $(e, f)=t(c, \pm d)$ for some non-zero scalar $t$. Moreover, $P_{3}(c, d)$ and $P_{3}(e, f)$ are congruent if and only if $(e, f)=( \pm c, \pm d)$.

Proof. The proof is very similar to that of Lemma 5.8. Suppose $S$ is an affine transformation with $P_{3}(e, f)=P_{3}(c, d) S, o S=o$ and $(c,-c, d) S=(e,-e, f)$. Since $S$ and $S^{-1}$ preserve planarity of vertex-stars, we certainly have $c=0$ if and only if $e=0$. If $c \neq 0$, we have non-planar vertex-stars and hence can conclude as in Lemma 4.7 that $S^{-1} T(c, d) S=T(e, f)$ and $S^{-1} S_{2} S=S_{2}$ or $S_{2}^{-1}$. Then either $S=t I$ or $S=t R$, with $R$ as in (6.13), for some scalar $t$. Hence $(e, f)=t(c, \pm d)$. Moreover, if $S$ is an isometry, then $t= \pm 1$. The converse is clear (by (6.12)).

Notice that the conditions of Lemmas 5.8 and 6.6 are the same, that is, $P_{3}(c, d)$ and $P_{3}(e, f)$ are affinely equivalent if and only if $P_{2}(c, d)$ and $P_{2}(e, f)$ are affinely equivalent. Thus the affine equivalence classes of polyhedra $P_{3}(c, d)$ can be illustrated in a similar fashion as those of $P_{2}(c, d)$ (see Fig. 6.2). At the corners of the square we now find the regular polyhedra $P_{3}(0,1)=\{\infty, 4\}{ }_{, * 3}$ and $P_{3}(1,0)=\{3,4\}$. As before, each equivalence class is represented by a polyhedron with its parameter point $(c, d)$ located on the upper right side of the square; the other three sides of the square give alternative representations.

The faces of $P_{3}(c, d)$ consist of either all right-hand helices or all left-hand helices, and the helices for $P_{3}(c, d)$ are right-hand if and only if those for $P_{3}(c,-d)\left(=P_{3}(c, d) R\right)$ are left-hand. Each face has its axis parallel to a body diagonal of $\mathbb{Z}^{3}$. The next lemma

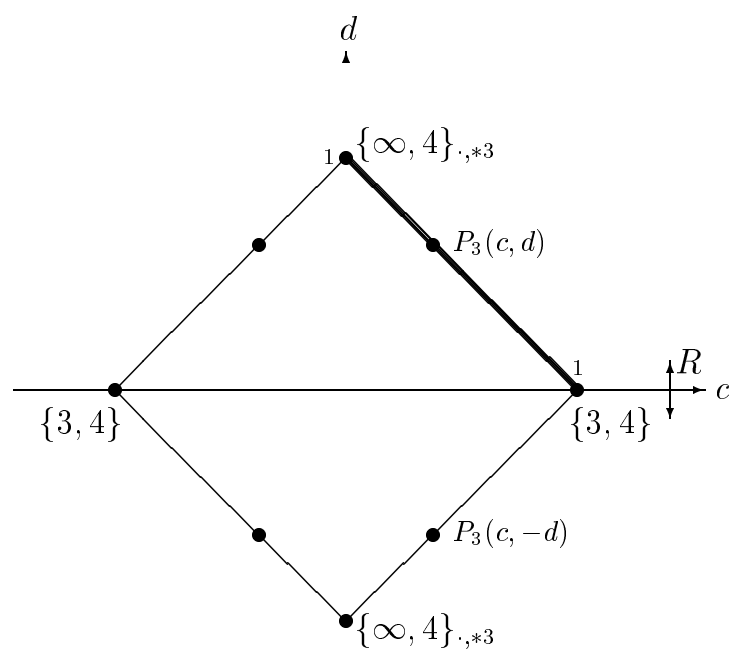

Fig. 6.2. The affine classes of polyhedra $P_{3}(c, d)$. 
describes the translation classes of faces modulo $d \Lambda_{(1,1,1)}$; in the regular case they are also the translation classes relative to the full symmetry group.

Lemma 6.7. $\quad P_{3}(c, d)$ has eight translation classes of faces, except when $c=0$ or $c= \pm d$; they are represented by the faces $F_{2} T_{1}^{i} S_{2}^{j}$ with $i=0,1$ and $j=0,1,2,3$, where $F_{2}$ is the base face given by (6.4). If $c=0$ or $c= \pm d$, then $P_{3}(c, d)$ has four translation classes of faces, represented by $F_{2} S_{2}^{j}$ with $j=0,1,2,3$.

Proof. We only outline the argument. Suppose $d \neq 0$. Each vertex of $P_{3}(c, d)$ is equivalent modulo $d \Lambda_{(1,1,1)}$ to one of the (generally six) vertices $o\left(T_{0} T_{1}\right)^{k} T_{0}^{m}$ with $k=$ $0,1,2$ and $m=0,1$ (see the proof of Lemma 6.2, particularly (6.8)), so each face must be equivalent to a face containing one of these vertices. This latter set of faces consists of the four faces $F_{2} S_{2}^{j}$ with $j=0,1,2,3$, each of which contains $o$ and is parallel to one of the four body diagonals of $\mathbb{Z}^{3}$, as well as their images under the six elements $U:=\left(T_{0} T_{1}\right)^{k} T_{0}^{m}$ with $k, m$ as above. The face $\widehat{F}_{2}:=F_{2} S_{2}^{3}$ has its axis parallel to the diagonal with direction vector $(-1,1,1)$, and since $T_{0}^{\prime}$ and $T_{1}^{\prime}$ (the images of $T_{0}$ and $T_{1}$ in the special group) reverse this direction, this also remains true for each face $\widehat{F}_{2} U$ with $U$ as above. Inspection shows that, for all parameter values of $c$ and $d$, these six faces fall into two sets of three, where each set consists of mutually equivalent faces modulo $d \Lambda_{(1,1,1)}$ (all $U$ with $m=0$, or all $U$ with $m=1$ ). Moreover, faces from different sets can only be equivalent if $c=0$ or $c= \pm d$; in fact, in these cases any two faces of the six are equivalent modulo $d \Lambda_{(1,1,1)}$. Hence, the faces parallel to the direction vector $(-1,1,1)$ fall into two classes or one class, respectively, and are represented by either $\widehat{F}_{2}, \widehat{F}_{2} T_{0}$ or $\widehat{F}_{2}$. This takes care of one diagonal direction. However, since the special group $G_{0}$ (in fact, already its subgroup $\left\langle S_{2}\right\rangle$ ) acts transitively on the four body diagonals, this also holds for other body diagonals, so we must have a total of eight or four translation classes, respectively. Finally, since

$$
\widehat{F}_{2} T_{0} S_{2}^{j+1}=F_{2}\left(S_{2}^{-1} T_{0} S_{2}\right) S_{2}^{j}=F_{2} T_{1} S_{2}^{j}
$$

(see (6.5)), the images of $\widehat{F}_{2} T_{0}$ under $\left\langle S_{2}\right\rangle$ are just those of $F_{2} T_{1}$ under $\left\langle S_{2}\right\rangle$, so the eight or four classes, respectively, are represented by the images under $\left\langle S_{2}\right\rangle$ of either $F_{2}, F_{2} T_{1}$ or $F_{2}$. This completes the proof.

\section{Coverings and Relationships}

In this and the next section we investigate coverings and relationships for the families of chiral (or regular) polyhedra described in the previous sections and [24]. Recall that we have three large families of chiral (or regular) polyhedra with finite faces, consisting of the polyhedra $P(a, b)$ of type $\{6,6\}, Q(c, d)$ of type $\{4,6\}$ or $Q(c, d)^{*}$ (the dual of $Q(c, d)$ ) of type $\{6,4\}$, respectively. In addition we have the three large families of chiral (or regular) helix-faced polyhedra $P_{1}(a, b), P_{2}(c, d)$ or $P_{3}(c, d)$ discussed in this paper.

We begin with the observation that the helix-faced polyhedra $P_{1}(a, b), P_{2}(c, d)$ and $P_{3}(c, d)$ can be thought of as figures "unraveling" a tetrahedron $\{3,3\}$, a cube $\{4,3\}$ or an octahedron $\{3,4\}$, respectively; that is, in a way, the helical faces can be pushed down 
to their bases, like springs, to become faces of $\{3,3\},\{4,3\}$ or $\{3,4\}$. The underlying covering relationship of polyhedra is induced by the canonical homomorphism

$$
\begin{aligned}
G & \rightarrow G_{0}(\cong G / T(G)), \\
S & \mapsto S^{\prime},
\end{aligned}
$$

which maps the distinguished generators $S_{1}, S_{2}$ of $G$ to those of $G_{0}$. For example, for $P_{1}(a, b)$ with group $G=G_{1}(a, b)=\left\langle S_{1}, S_{2}\right\rangle$, the images $S_{1}^{\prime}, S_{2}^{\prime}\left(=S_{2}\right)$ are distinguished generators of $G_{0}=[3,3]^{+}$. Thus we have a covering $P_{1}(a, b) \searrow\{3,3\}$ (see Section 2D of [20]).

Theorem 7.1. There are the following coverings of polyhedra:

$$
P_{1}(a, b) \searrow\{3,3\}, \quad P_{2}(c, d) \searrow\{4,3\}, \quad P_{3}(c, d) \searrow\{3,4\} .
$$

In each case the polyhedron on the right is the only finite polyhedron in its family and is obtained for $b=a, c=0$ or $d=0$, respectively.

In a similar fashion we can also derive the coverings

$$
Q(c, d) \searrow\{4,6 \mid \cdot, 2\}, \quad Q(c, d)^{*} \searrow\{4,6 \mid \cdot, 2\}^{*},
$$

where $\{4,6 \mid \cdot, 2\}$ denotes the regular map of type $\{4,6\}$ determined by the length, 2 , of its 3-holes (the 2-holes are not needed for the specification). Recall that a 3-hole is an edge-path which leaves a vertex by the third edge from which it entered, always keeping to the right or always to the left (see p. 196 of [20]). In fact, for $Q(c, d)$ and its dual, the special group $G_{0}(=[3,4])$ admits an involutory group automorphism with $S_{1}^{\prime} \mapsto S_{1}^{\prime} S_{2}^{\prime 2}$ and $S_{2}^{\prime} \mapsto S_{2}^{\prime-1}$, so the abstract polyhedron $\mathcal{Q}^{0}$ associated with $G_{0}$ and its generators $S_{1}^{\prime}, S_{2}^{\prime}$ must actually be directly regular, with full automorphism group $G_{0} \rtimes C_{2}$ (see Theorem 1(c) of [25]). (Recall that an abstract polyhedron is directly regular if it is regular and its combinatorial rotation subgroup has index 2 in the full automorphism group. The corresponding surface is necessarily orientable.) For $Q(c, d)$, this group automorphism can be realized by (inner) conjugation in $G_{0}$ with the reflection in the plane $\xi_{1}=\xi_{2}$ (see (6.17) of [24]). In particular, since $\mathcal{Q}^{0}$ is orientable and of genus 3 , we must have $\mathcal{Q}^{0}=\{4,6 \mid \cdot, 2\}$ (see p. 470 of [28]).

Note, however, that there is no such covering associated with the polyhedra $P(a, b)$. The generators $S_{1}^{\prime}, S_{2}^{\prime}$ of $G_{0}\left(=[3,3]^{*}\right)$ do not determine a (directly) regular or chiral map in this case; in fact, $-I \in\left\langle S_{1}^{\prime}\right\rangle \cap\left\langle S_{2}^{\prime}\right\rangle$, so the intersection property fails.

Next we study operations between polyhedra or their groups. Recall that a mixing operation derives a new group $H$ from a given group $G=\left\langle S_{1}, S_{2}\right\rangle$ by taking as generators $\widehat{S}_{1}, \widehat{S}_{2}$ (say) certain suitably chosen products of $S_{1}, S_{2}$, so that $H$ is a subgroup of $G$ (see p. 183 of [20]). Mixing operations do not always produce groups of chiral or regular polyhedra, but in certain special cases they do. Besides the duality operation $\delta$ and the facetting operation $\varphi_{2}$ described in (2.2), we also employ the halving operation

$$
\eta:\left(S_{1}, S_{2}\right) \mapsto\left(S_{1}^{2} S_{2}, S_{2}^{-1}\right)=:\left(\widehat{S}_{1}, \widehat{S}_{2}\right)
$$


(see Section 7 of [24]), which is an analog of the halving operation for regular polyhedra (see p. 197 of [20]). Recall from Section 7 of [24] that $\eta$ links the two families of finite-faced polyhedra $Q(c, d)$ and $P(a, b)$ through

$$
Q(c, d)^{\eta} \cong P(c-d, c+d),
$$

where $Q(c, d)^{\eta}$ denotes the image of $Q(c, d)$ under $\eta$ (and $\cong$ means congruence). We also observed in Lemmas 4.1 and 5.1 that

$$
P_{1}(a, b)=P(a, b)^{\varphi_{2}}, \quad P_{2}(c, d)=Q(c, d)^{\varphi_{2}} .
$$

Generally it is difficult to decide whether or not two classes of polyhedra are related by a mixing operation. In some cases obstructions already arise from the structure of the special groups. In fact, we have the following lemma.

Lemma 7.2. Let $G$ and $H$ be crystallographic groups with special groups $G_{0}$ and $H_{0}$, respectively. If $H$ is a subgroup of $G$, then $H_{0}$ is isomorphic to a subgroup of $G_{0}$.

Proof. If $H \leqslant G$, then the mapping $H \rightarrow G / T(G)$ defined by $S \mapsto T(G) S$ has kernel $H \cap T(G)=T(H)$, so $H / T(H)$ is isomorphic to a subgroup of $G / T(G)$. Now the lemma follows.

For example, the special groups tell us immediately that $P_{3}(c, d)$ cannot be derived from a mixing operation applied to a polyhedron $P(a, b)$ (see Section 5 of [24]). In fact, by the previous lemma, $G_{3}(c, d)$ cannot be a subgroup of $G(a, b)$ (the group for $P(a, b))$, because clearly $\left(G_{3}(c, d)\right)_{0}\left(=[3,4]^{+} \cong S_{4}\right)$ is not isomorphic to a subgroup of $(G(a, b))_{0}\left(=[3,3]^{*} \cong A_{4} \times C_{2}\right)$. Similarly we can eliminate the possibility that $P_{3}(c, d)$ is obtained from a mixing operation applied to a helix-faced polyhedron $P_{1}(a, b)$ (with special group [3.3 $]^{+} \cong A_{4}$ ).

On the other hand, we cannot, a priori, rule out a mixing operation between $P_{3}(c, d)$ and a polyhedron $Q\left(c^{\prime}, d^{\prime}\right)$ or its dual; in fact, $Q\left(c^{\prime}, d^{\prime}\right)$ and its dual have special group $[3,4]\left(\cong S_{4} \times C_{2}\right)$. The same remark also applies to $P_{3}(c, d)$ and a polyhedron $P_{2}\left(c^{\prime}, d^{\prime}\right)$ (with special group $[3,4]^{+}$). In these cases it is open whether or not mixing operations exist. On the other hand, we do know that the regular polyhedron $P_{3}(0,1)=\{\infty, 4\}_{, * 3}$ is obtained from the Petrie-Coxeter polyhedron $Q(0,1)^{*}=\{6,4 \mid 4\}$ by the skewing (mixing) operation $\sigma$ defined on pp. 199 and 224 of [20]; that is, $P_{3}(0,1)=\left(Q(0,1)^{*}\right)^{\sigma}$. However, $\sigma$ is only defined for regular polyhedra, and it is not clear how to generalize it to chiral polyhedra.

In the next section we describe the missing link between $P_{3}(c, d)$ and a finitefaced polyhedron, namely $Q(-d, 2 c)^{*}$, employing an involutory operation $\kappa$ which is not a mixing operation on its group (see Lemma 8.1). When applied to $P(a, b)$ and $Q(c, d)$, this operation $\kappa$ coincides, up to the enantiomorphism, with the facetting operation $\varphi_{2}$.

We now list the families of polyhedra, with various known relationships between them (but see also (8.3)). Our first diagram emphasizes operations between families rather than between individual polyhedra. In particular, we drop the parameters from the notation; 
for example, $P_{1}$ denotes the family of polyhedra $P_{1}(a, b)$. Observe that $P_{3}$ is connected to $Q^{*}$ via $\kappa$. The circular arrow indicates the self-duality of the family (or polyhedron).

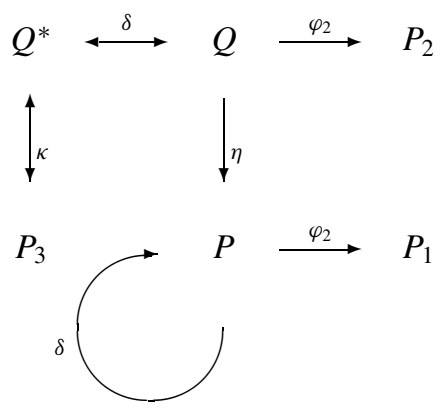

The corresponding parameters can be read off the next diagram for (congruence classes of) individual polyhedra; here, $Q(c, d)$ is the reference point.

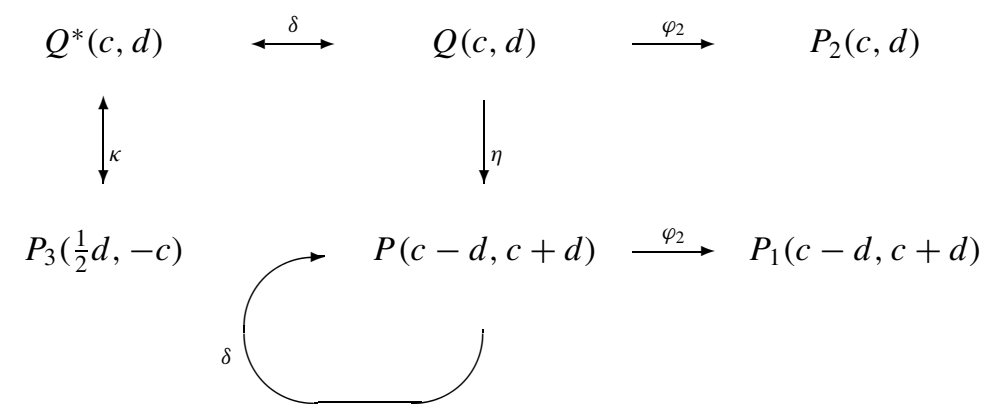

It is instructive to list the families of polyhedra by the structure of their special group, along with the two regular polyhedra occurring in each family.

\begin{tabular}{cccccc}
\hline$[3,3]^{*}$ & {$[4,3]$} & {$[3,4]$} & {$[3,3]^{+}$} & {$[4,3]^{+}$} & {$[3,4]^{+}$} \\
\hline$P(a, b)$ & $Q(c, d)$ & $Q(c, d)^{*}$ & $P_{1}(a, b)$ & $P_{2}(c, d)$ & $P_{3}(c, d)$ \\
\hline$\{6,6\}_{4}$ & $\{4,6\}_{6}$ & $\{6,4\}_{6}$ & $\{\infty, 3\}^{(a)}$ & $\{\infty, 3\}^{(b)}$ & $\{\infty, 4\}_{, * 3}$ \\
$\{6,6 \mid 3\}$ & $\{4,6 \mid 4\}$ & $\{6,4 \mid 4\}$ & $\{3,3\}$ & $\{4,3\}$ & $\{3,4\}$ \\
\hline
\end{tabular}

In this table the columns are indexed by the special groups to which the respective polyhedra correspond. The second row contains the families. The last two rows comprise nine of the twelve discrete pure regular polyhedra in $\mathbb{E}^{3}$, namely those listed in the table on p. 225 of [20] with dimension vectors

$$
\left(\operatorname{dim} R_{0}, \operatorname{dim} R_{1}, \operatorname{dim} R_{2}\right)=(1,2,1),(1,1,1),(2,1,2),
$$

as well as the three (finite) "crystallographic" Platonic polyhedra. The three remaining pure regular polyhedra $\{\infty, 6\}_{4,4},\{\infty, 4\}_{6,4}$ and $\{\infty, 6\}_{6,3}$ have dimension vector $(1,1,2)$ 
and do not occur in families alongside chiral polyhedra (see Lemma 3.3). Note that $\varphi_{2}$, when applicable, and $\kappa$ map a polyhedron to one in the same row.

A final comment about the regular polyhedra $P=\{\infty, 3\}^{(a)},\{\infty, 3\}^{(b)}$ or $\{\infty, 4\}_{, * 3}$ is appropriate. In each case the full symmetry group $G(P)$ is generated by half-turns $R_{0}, R_{1}, R_{2}$, so the special group $G(P)_{0}$ consists only of rotations and hence coincides with $G_{0}$. The generators $R_{0}^{\prime}, R_{1}^{\prime}, R_{2}^{\prime}$ of $G(P)_{0}$ are also half-turns. For example, if $P=\{\infty, 4\}_{, * * 3}$, then $G(P)_{0}=G_{0}=[3,4]^{+}$(viewed as the rotation group of an octahedron) and the rotation axes of $R_{0}^{\prime}, R_{1}^{\prime}$ or $R_{2}^{\prime}$ are the lines through $o$ and $(-1,1,0)$, $(-1,0,1)$ or $(0,0,1)$, respectively. It is not difficult to see that, in each case, $G(P)_{0}$ is a C-group with respect to these generators (see Section 2E of [20]), so there exists a finite abstract regular polyhedron $\mathcal{P}^{0}$ with automorphism group $G(P)_{0}$ and distinguished generators $R_{0}^{\prime}, R_{1}^{\prime}, R_{2}^{\prime}$. (The abstract polyhedron $\mathcal{Q}^{0}$ mentioned earlier arises in a similar way from $\{4,6 \mid 4\}=Q(0,1)$.) Inspection shows that $\mathcal{P}^{0}=\{3,3\},\{4,3\}_{3}$ or $\{3,4\}_{3}$, respectively. However, except in the first case, $\mathcal{P}^{0}$ does not coincide with the regular polyhedron determined by $G_{0}$ and its generators $S_{1}^{\prime}, S_{2}^{\prime}$; we know the latter to be $\{3,3\},\{4,3\}$ and $\{3,4\}$, respectively. In fact, only when $\mathcal{P}^{0}$ is directly regular should we expect isomorphism for these polyhedra (see p. 510 of [25]). On the other hand, the very fact that conjugation by $R_{2}^{\prime}$ yields an involutory group automorphism of $G_{0}$ with $S_{1}^{\prime} \mapsto S_{1}^{\prime} S_{2}^{\prime 2}$ and $S_{2}^{\prime} \mapsto S_{2}^{\prime-1}$, correctly predicts that the polyhedron associated with $G_{0}$ and $S_{1}^{\prime}, S_{2}^{\prime}$ is directly regular and has group $G_{0} \rtimes C_{2}$. (Since $\{\infty, 3\}^{(a)}$ and $\{\infty, 3\}^{(b)}$ are Petrie-duals, the same is true for their respective polyhedra $\mathcal{P}^{0}$, namely $\{3,3\}$ and $\{4,3\}_{3}$. Moreover, since $\{6,4 \mid 4\}^{\sigma}=\{\infty, 4\}_{, * * 3}$, we also have $\left(\{4,6 \mid \cdot, 2\}^{*}\right)^{\sigma}=\{3,4\}_{3}$, which is self-Petrie.)

Notice, however, that $\mathcal{P}^{0}$ does not correspond to a geometrically regular polyhedron; in fact, no point (distinct from $o$ ) is admissible as the initial vertex for Wythoff's construction applied to $G(P)_{0}$ and $R_{0}^{\prime}, R_{1}^{\prime}, R_{2}^{\prime}$.

These considerations also shed some light on why there are exactly two regular polyhedra in each of the six families. In fact, given a family with special group $G_{0}$, there are precisely two involutory isometries $R$ such that conjugation by $R$ determines a group automorphism of $G_{0}$ with $S_{1}^{\prime} \mapsto S_{1}^{\prime} S_{2}^{\prime 2}$ and $S_{2}^{\prime} \mapsto S_{2}^{\prime-1}$ (and hence $T^{\prime} \mapsto T^{\prime}$ ). Each $R$ is the image $R_{2}^{\prime}$, in $G_{0}$, of the generator $R_{2}$ for a regular polyhedron in the same family. For example, if $P=\{\infty, 4\}_{, * 33}$, then $S_{1}^{\prime}, S_{2}^{\prime}\left(=S_{2}\right)$ are as in (6.1) and $R$ is either the half-turn about the $\xi_{3}$-axis or the reflection in the $\xi_{1} \xi_{2}$-plane (the two isometries $R$ have perpendicular mirrors). The first choice of $R$ leads to $P_{3}(0,1)=$ $\{\infty, 4\}_{\cdot, * 3}$ (with $R_{0}^{\prime}, R_{1}^{\prime}, R_{2}^{\prime}$ as above), and the second to $P_{3}(1,0)=\{3,4\}$. For the three families $P_{1}(a, b), P_{2}(c, d)$ and $P_{3}(c, d)$ of helix-faced polyhedra, $R$ is either the reflection in the plane spanned by the rotation axes of $T^{\prime}$ and $S_{2}^{\prime}$, or the half-turn about the line perpendicular to this plane at $o$. The same remains true for the three families $P(a, b), Q(c, d)$ and $Q(c, d)^{*}$ of finite-faced polyhedra, except that here $S_{2}^{\prime}$ is replaced by $S_{2}^{\prime 2}$.

\section{Connecting $P_{3}(c, d)$}

In this final section we briefly discuss an operation that establishes the missing link between $P_{3}(c, d)$ and the polyhedra with finite faces. This observation is due to Peter 
McMullen and is reproduced here with his permission. Similar operations were employed in [17] for the classification of regular polytopes of full rank.

Let $P$ be any chiral (or regular) polyhedron with corresponding group $G=\left\langle S_{1}, S_{2}\right\rangle$. Assume, as usual, that the initial vertex (fixed by $S_{2}$ ) is $o$. Define $K:=-I$ to be the reflection in $o$. Now consider the operation

$$
\kappa:\left(S_{1}, S_{2}\right) \mapsto\left(S_{1} K, K S_{2}\right)=:\left(\tilde{S}_{1}, \tilde{S}_{2}\right)
$$

We show that this involutory operation interchanges the class of finite-faced polyhedra with the class of helix-faced polyhedra. Observe that $\kappa$ preserves $T:=S_{1} S_{2}$; that is, if $\tilde{T}:=\tilde{S}_{1} \tilde{S}_{2}$, then $\tilde{T}=T$. (Note also that, since $K$ commutes with $S_{2}$, we could have replaced $S_{1} K$ by $K S_{1}$ to obtain a conjugate group.)

Lemma 8.1. The involutory operation $\kappa$ pairs up polyhedra as follows:

(a) $P_{1}(a, b)=P(a, b)^{\kappa}$, for all real parameters $a$ and $b$.

(b) $P_{2}(c, d)=Q(c, d)^{\kappa}$, for all real parameters $c$ and $d$.

(c) $P_{3}(c, d) \cong\left(Q(-d, 2 c)^{*}\right)^{\kappa}$, for all real parameters $c$ and $d$.

Proof. When $P$ is of type $\{6,6\}$ or $\{4,6\}$ (that is, when $S_{2}$ has period 6), if we follow $\kappa$ by the enantiomorphism operation $\mu$ of (2.3), and recall from [24] that $K=S_{2}^{3}$, we obtain

$$
\left(S_{1}, S_{2}\right) \mapsto\left(\tilde{S}_{1} \tilde{S}_{2}^{2}, \tilde{S}_{2}^{-1}\right)=\left(S_{1} S_{2}^{3} \cdot S_{2}^{8}, S_{2}^{-4}\right)=\left(S_{1} S_{2}^{-1}, S_{2}^{2}\right),
$$

which is just the facetting operation $\varphi_{2}$; that is, $\kappa \mu=\varphi_{2}$. In other words, $P^{\kappa}=P^{\varphi_{2}}$; in particular, $P^{\kappa}$ and $P^{\varphi_{2}}$ represent the two enantiomorphic forms of the same underlying polyhedron. Now Lemmas 4.1 and 5.1 prove parts (a) and (b).

For part (c) the analysis is more elaborate. Now let $P:=P_{3}(c, d)$ and follow $\kappa$ by the duality operation $\delta$ (employing $\delta$ allows a direct comparison with generators used in [24]). Then the generators $S_{1}$ and $S_{2}$ of (6.2) and (6.3) for $P$ yield those for $\left(P^{\kappa}\right)^{*}$, namely

$$
\begin{array}{llll}
\widehat{S}_{1}:=\tilde{S}_{2}^{-1}: & x & \mapsto \quad\left(\xi_{3},-\xi_{2},-\xi_{1}\right), \\
\widehat{S}_{2}:=\tilde{S}_{1}^{-1}: & x \quad \mapsto \quad-\left(\xi_{2}, \xi_{3}, \xi_{1}\right)+(c,-c, d) .
\end{array}
$$

The fixed point of $\widehat{S}_{2}$ is

$$
z:=\left(c+\frac{1}{2} d,-\frac{1}{2} d,-c+\frac{1}{2} d\right)
$$

(not $o$ ). The direction vector of the base edge of $\left(P^{\kappa}\right)^{*}$ is given by

$$
z \widehat{T}-z=(-d, d, 2 c),
$$

where $\widehat{T}:=\widehat{S}_{1} \widehat{S}_{2}$, so we should expect the parameters to be $-d, 2 c$, not $c, d$. We now establish congruence of $\left(P^{\kappa}\right)^{*}$ with the polyhedron $Q(-d, 2 c)$ as follows. Begin with the distinguished generators of the group for $Q(-d, 2 c)$ described in (6.1) and (6.2) of [24] (denoted by $S_{1}, S_{2}$ ), and replace them by the alternative pair $U_{1}, U_{2}$ of generators 
under enantiomorphism. It is straightforward to check that if $R$ denotes the translation by $z$, then

$$
R^{-1} U_{1} R=\widehat{S}_{1}, \quad R^{-1} U_{2} R=\widehat{S}_{2} .
$$

It follows that $\left(P^{\kappa}\right)^{*}=Q(-d, 2 c) R$ and hence $P^{\kappa}=Q(-d, 2 c)^{*} R$. In particular, $P^{\kappa}=Q(-d, 2 c)^{*}$, up to translation, and therefore also $P=\left(Q(-d, 2 c)^{*}\right)^{\kappa}$, up to translation.

We summarize the relationships in the following diagram; the corresponding parameters can be read off diagram (7.4) (with $\varphi_{2}$ replaced by $\kappa$ ):

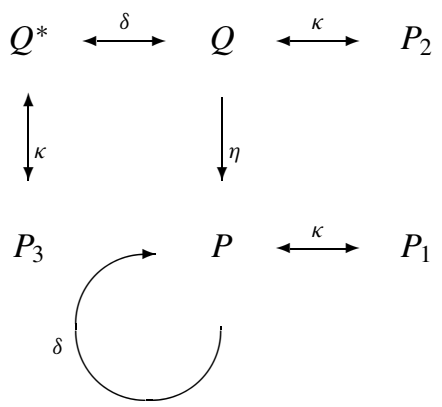

The two arrows for $\kappa$ on the right in (8.3) are double arrows, although those for $\varphi_{2}$ in diagram (7.3) are not. None of the helix-faced polyhedra is centrally symmetric with respect to a vertex, so in particular $K$ is not contained in its group. It follows that $\kappa$ (applied to one of them) is generally not a mixing operation. However, for $P(a, b)$ and $Q(c, d)$ we do obtain a mixing operation $\kappa$ (namely $\varphi_{2} \mu$ ). Notice, however, that the groups of $P(a, b)^{\kappa}$ and $Q(c, d)^{\kappa}$ are proper subgroups of those of $P(a, b)$ and $Q(c, d)$, respectively, even though $\kappa$ is involutory.

One last comment is appropriate. The special group of a finite-faced polyhedron is generated by rotatory reflections, while the special group of a helix-faced polyhedron is generated by rotations. When applied to the special groups, $\kappa$ turns generating rotatory reflections into generating rotations, and vice versa.

\section{Acknowledgment}

Finally, as a birthday greeting, I wish to acknowledge the deep influence Branko Grünbaum's work has had on my research over the years. His pioneering papers [11], [12] on generalized geometric or abstract regular polyhedra or polytopes lie at the heart of the enumeration presented here.

\section{References}

1. L. Bieberbach, Über die Bewegungsgruppen der euklidischen Räume: erste Abhandlung, Math. Ann. 70 (1910), 297-336.

2. U. Brehm and J.M. Wills, Polyhedral manifolds, in Handbook of Convex Geometry (eds. P.M. Gruber and J.M. Wills), Elsevier, Amsterdam, 1993, pp. 535-554. 
3. L. Chen, R.V. Moody and J. Patera, Non-crystallographic root systems, in Quasicrystals and Discrete Geometry (ed. J. Patera), Fields Institute Monographs, Vol. 10, American Mathematical Society, Providence, RI, 1998, pp. 135-178.

4. J.H. Conway and N.J.A. Sloane, Sphere Packings, Lattices and Groups. Springer-Verlag, New York, 1988.

5. H.S.M. Coxeter, Regular skew polyhedra in 3 and 4 dimensions and their topological analogues, Proc. London Math. Soc. (2) 43 (1937), 33-62. (Reprinted with amendments in Twelve Geometric Essays, Southern Illinois University Press, Carbondale, IL, 1968, pp. 76-105.)

6. H.S.M. Coxeter, Regular Polytopes (3rd edition), Dover, New York, 1973.

7. H.S.M. Coxeter and W.O.J. Moser, Generators and Relations for Discrete Groups (4th edition), SpringerVerlag, New York, 1980

8. A.W.M. Dress, A combinatorial theory of Grünbaum's new regular polyhedra, I: Grünbaum's new regular polyhedra and their automorphism group, Aequationes Math. 23 (1981), 252-265.

9. A.W.M. Dress, A combinatorial theory of Grünbaum's new regular polyhedra, II: complete enumeration, Aequationes Math. 29 (1985), 222-243.

10. L.C. Grove and C.T. Benson, Finite Reflection Groups (2nd edition), Graduate Texts in Mathematics 99, Springer-Verlag, New York, 1985.

11. B. Grünbaum, Regular polyhedra—old and new, Aequationes Math. 16 (1977), 1-20.

12. B. Grünbaum, Regularity of graphs, complexes and designs, in Problèmes combinatoires et théorie des graphes, Colloq. Internat. C.N.R.S. 260, CNRS, Paris, 1978, pp. 191-197.

13. B. Grünbaum, Polyhedra with hollow faces, in Polytopes: Abstract, Convex and Computational (eds. T. Bisztriczky, P. McMullen, R. Schneider and A. Ivić Weiss), NATO ASI Series C 440, Kluwer, Dordrecht, 1994, pp. 43-70.

14. B. Grünbaum, Acoptic polyhedra, in Advances in Discrete and Computational Geometry (eds. B.Chazelle et al.), Contemporary Mathematics 223, American Mathematical Society, Providence, RI, 1999, pp. 163199.

15. B. Grünbaum, Are your polyhedra the same as my polyhedra?, in Discrete and Computational Geometry, Algorithms and Combinatorics 25, Springer, Berlin, 2003, pp. 461-488.

16. Ch. Leytem, Regular polyhedra with translational symmetries, Period. Math. Hungar. 34 (1997), 111-122.

17. P. McMullen, Regular polytopes of full rank, Discrete Comput. Geometry 32 (2004), 1-35.

18. P. McMullen, Four-dimensional regular polyhedra. (In preparation.)

19. P. McMullen and E. Schulte, Regular polytopes in ordinary space, Discrete Comput. Geom. 17 (1997), 449-478.

20. P. McMullen and E. Schulte, Abstract Regular Polytopes, Encyclopedia of Mathematics and its Applications 92, Cambridge University Press, Cambridge, 2002.

21. P. McMullen and E. Schulte, Regular and chiral polytopes in low dimensions, in The Coxeter LegacyReflections and Projections (eds. C. Davis and E.W. Ellers), Fields Institute Communications, Vol. 48, American Mathematical Society Providence, RI, 2005, to appear.

22. B.R. Monson and A.I. Weiss, Realizations of regular toroidal maps, Canad. J. Math. 51(6) (1999), 12401257.

23. J.G. Ratcliffe, Foundations of Hyperbolic Manifolds. Graduate Texts in Mathematics 149, Springer-Verlag, New York, 1994.

24. E. Schulte, Chiral polyhedra in ordinary space, I, Discrete Comput. Geometry 32 (2004), 55-99.

25. E. Schulte and A.I. Weiss, Chiral polytopes, in Applied Geometry and Discrete Mathematics (The Victor Klee Festschrift) (eds. P. Gritzmann and B. Sturmfels), DIMACS Series in Discrete Mathematics and Theoretical Computer Science 4, American Mathematical Society and Assocociation of Computing Machinery, Providence, RI, and New York, 1991, pp. 493-516.

26. E. Schulte and A.I. Weiss, Chirality and projective linear groups, Discrete Math. 131 (1994), 221-261.

27. M. Senechal, Quasicrystals and Geometry, Cambridge University Press, Cambridge, 1995.

28. F.A. Sherk, The regular maps on a surface of genus three, Canad. J. Math. 11 (1959), 452-480.

29. J.M. Wills, Combinatorially regular polyhedra of index 2, Aequationes Math. 34 (1987), 206-220.

30. S.E. Wilson, New techniques for the construction of regular maps, Doctoral Dissertation, University of Washington, 1976.

Received December 22, 2004, and in revised form March 9, 2005. Online publication May 27, 2005. 